ground state of $\mathrm{Rb}^{88}$, the value for the average energy might be used to distinguish between various assumptions regarding the angular distribution of the electron and the neutrino. Should, however, $\beta$-decay to some intermediate state, followed by $\gamma$-radiation, occur, a correction must be applied to the measured value of the average energy to obtain the value corresponding to a simple $\beta$-spectrum. This correction may change the average recoil energy to such an extent that with our present lack of knowledge about the level scheme of the transformation, such comparisons would be inconclusive.

Our thanks are due to Professor Niels Bohr for his kind interest in our work, to Mr. N. O. Lassen and Mr. K. O. Nielsen for their help in the work with the cyclotron, to Mr. B. S. Madsen for the construction of the counters and to $\mathrm{Mr}$. J. Lindhard for some theoretical discussions.

\title{
Relaxation Effects in Nuclear Magnetic Resonance Absorption*
}

\author{
N. Bloembergen, ${ }^{* *}$ E. M. Purcell, and R. V. Pound, ${ }^{* * *}$ \\ Lyman Laboratory of Physics, Harvard University, Cambridge, Massachusetts
}

(Received December 29, 1947)

The exchange of energy between a system of nuclear spins immersed in a strong magnetic field, and the heat reservoir consisting of the other degrees of freedom (the "lattice") of the substance containing the magnetic nuclei, serves to bring the spin system into equilibrium at a finite temperature. In this condition the system can absorb energy from an applied radiofrequency field. With the absorption of energy, however, the spin temperature tends to rise and the rate of absorption to decrease. Through this "saturation" effect, and in some cases by a more direct method, the spin-lattice relaxation time $T_{1}$ can be measured. The interaction among the magnetic nuclei, with which a characteristic time $T_{2}{ }^{\prime}$ is associated, contributes to the width of the absorption line. Both interactions have been studied in a variety of substances, but with the emphasis on liquids containing hydrogen.

Magnetic resonance absorption is observed by means of a radiofrequency bridge; the magnetic field at the sample is modulated at a low frequency. A detailed analysis of the method by which $T_{1}$ is derived from saturation experiments is given. Relaxation times observed range from $10^{-4}$ to $10^{2}$ seconds. In liquids $T_{1}$ ordinarily decreases with increasing viscosity, in some cases reaching a minimum value after which it increases with further increase in viscosity. The line width meanwhile increases monotonically from an extremely small value toward a value determined by the spin-spin interaction in the rigid lattice.

\section{INTRODUCTION}

$I^{\mathrm{N}}$ nuclear magnetic resonance absorption, energy is transferred from a radiofrequency

* A brief account of this work has appeared in Nature 160,475 (1947).

** Present address: Kamerlingh Onnes Laboratory, University of Leiden.

*** Society of Fellows.
The effect of paramagnetic ions in solution upon the proton relaxation time and line width has been investigated. The relaxation time and line width in ice have been measured at various temperatures.

The results can be explained by a theory which takes into account the effect of the thermal motion of the magnetic nuclei upon the spin-spin interaction. The local magnetic field produced at one nucleus by neighboring magnetic nuclei, or even by electronic magnetic moments of paramagnetic ions, is spread out into a spectrum extending to frequencies of the order of $1 / \tau_{c}$, where $\tau_{c}$ is a correlation time associated with the local Brownian motion and closely related to the characteristic time which occurs in Debye's theory of polar liquids. If the nuclear Larmor frequency $\omega$ is much less than $1 / \tau_{c}$, the perturbations caused by the local field nearly average out, $T_{1}$ is inversely proportional to $\tau_{c}$, and the width of the resonance line, in frequency, is about $1 / T_{1}$. A similar situation is found in hydrogen gas where $\tau_{c}$ is the time between collisions. In very viscous liquids and in some solids where $\omega \tau_{c}>1$, a quite different behavior is predicted, and observed. Values of $\tau_{c}$ for ice, inferred from nuclear relaxation measurements, correlate well with dielectric dispersion data.

Formulas useful in estimating the detectability of magnetic resonance absorption in various cases are derived in the appendix.

circuit to a system of nuclear spins immersed in a magnetic field, $H_{0}$, as a result of transitions among the energy levels of the spin system. For each of $N$ non-interacting spins, characterized in the usual way by $I \hbar$ and $\mu$, the maximum $z$ components of angular momentum and magnetic moment, respectively, there are $2 I+1$ 
levels spaced in energy by $\mu H_{0} / I$. The state of this (non-interacting system) can be described by the magnetic quantum numbers $m_{1} \cdots m_{i}$ $\cdots m_{N}$, where $-I<m_{i} \ll I$. Upon the application of a suitable oscillating magnetic field, transitions corresponding to stimulated emission $\left(\Delta m_{i}=+1\right)$ and to absorption $\left(\Delta m_{i}=-1\right)$ will occur. If transitions of the latter sort are to preponderate, so that there is a net absorption of energy from the radiation field, it is essential that there be initially a surplus of spins in the lower states. This condition will be attained eventually if there is some way by which the spin system can interact with its surroundings and come to thermal equilibrium at a finite temperature. At equilibrium the population of the $2 I+1$ levels will be governed by the Boltzmann factor $\exp \left(\mu H_{0} m_{i} / I k T\right)$, and the requisite surplus in lower states will have been established.

The exposure of the system to radiation, with consequent absorption of energy, tends to upset the equilibrium state previously attained, by equalizing the population of the various levels. The new equilibrium state in the presence of the radiofrequency field represents a balance between the processes of absorption of energy by the spins, from the radiation field, and the transfer of energy to the heat reservoir comprising all other internal degrees of freedom of the substance containing the nuclei in question. The latter process involves what we shall call for short the spin-lattice interaction, and is described by a characteristic time, $T_{1}$, the spin-lattice relaxation time. It is this time also which measures how long one must wait, after the application of the constant field $H_{0}$, for the establishment of thermal equilibrium.

It should be evident that the above-mentioned competition between resonance absorption and spin-lattice interaction provides a way of measuring $T_{1}$. In a precisely similar way one might study the heat transfer between a wire and a surrounding bath by examining the temperature of the wire as a function of the power dissipated in the wire because of a current flowing through it. In our case the resonance absorption itself supplies a convenient thermometer, for the intensity of the absorption, depending as it does upon the surplus of spins in lower states, reflects the "temperature" of the spin system. An equally direct method, in the case of the wire, would be the measurement of the temperature of the wire, as a function of time, after switching off the heating current. The exact nuclear absorption analog of this experiment has also been carried out, and affords a striking manifestation of the relaxation process.

It may be said at once that nuclear spin-lattice relaxation times appear to range between $10^{-4}$ and $10^{2}$ seconds. Perhaps it seems remarkable at first that times so long should be associated with any atomic process. On the contrary, such times were, from the theoretical point of view, unexpectedly short, for the processes which had been examined theoretically ${ }^{1}$ prior to the first experiments in this field yielded much longer relaxation times. In the first experimental detection of nuclear resonance absorption in a solid, ${ }^{2}$ it was shown that the relaxation time for protons in paraffin, at room temperature and 7000 gauss, was certainly less than one minute. Subsequent work has shown that $T_{1}$ for this substance, under those conditions, is in fact very much shorter. Additional information on nuclear relaxation times has come from the work of Bloch and collaborators, ${ }^{3}$ and of Rollin ${ }^{4,5}$ who was the first to investigate the phenomenon at very low temperatures. The work here reported began as a more or less cursory survey of typical substances and grew, as the theoretical interpretation of the results progressed, into a systematic study of certain types of structures which forms the basis for a comprehensive theory of nuclear magnetic relaxation applicable to liquids, some gases, and certain types of solids.

It is obvious that the model of non-interacting spins described above is incomplete, for there will necessarily be interaction among the spins due to the associated nuclear magnetic moments, not to mention other possible sources of magnetic interaction such as electronic states of nonvanishing magnetic moment. We shall continue

\footnotetext{
${ }^{1}$ W. Heitler and E. Teller, Proc. Roy. Soc. 155A, 629 (1936).

${ }^{2}$ E. M. Purcell, H. C. Torrey, and R. V. Pound, Phys. Rev. 69, 37 (1946).

${ }^{3}$ F. Bloch, W. W. Hansen, and M. Packard, Phys. Rev. 70, 474 (1946).

4 B. V. Rollin, Nature 158, 669 (1946); B. V. Rollin and J. Hatton, Nature 159, 201 (1947).

${ }^{5}$ B. V. Rollin, J. Hatton, A. H. Cooke, and R. J. Benzie, Nature 160, 457 (1947).
} 
temporarily to ignore interactions of the latter sort, as well as possible electric quadrupole effects -it will turn out that we are justified in doing so for a sufficiently important class of substances - but we must certainly take into account the nuclear dipole-dipole interaction.

The interaction energy of neighboring dipoles separated by the distance $r$ is $\mu^{2} / r^{3}$ in order of magnitude, and if $H_{0} \mu \gg \mu^{2} / r^{3}$ we may expect, since more remote neighbors are relatively ineffective, that the result of the interactions will be a broadening of each of the $2 I+1$ levels mentioned earlier. From a point of view which is somewhat naive but useful within limits, we may say that the total magnetic field in the $z$ direction at the $i$ th nucleus is $H_{0}$ plus a "local" field $H_{\text {loc }}$ of order of magnitude

$$
\left|H_{\text {loc }}\right| \approx(\mu / I) \sum_{j} m_{j} r_{i j}{ }^{-3}
$$

and that the resonance condition for a transition $\Delta m_{i}= \pm 1$ is $h \nu=\left(H_{0}+H_{\mathrm{loc}}\right) \mu / I$. One would thus be led to expect the width of the absorption line, expressed in gauss, to be of the order $\mu / r^{3}$. Taking another point of view, a spin $j$ causes at its neighbor, $i$, an oscillating field at the resonance frequency, because of its own precession about $H_{0}$. This field is capable of inducing transitions in which $i$ and $j$ exchange energy. The lifetime against transitions is about $\hbar r^{3} / \mu^{2}$, leading again to a line width, expressed in gauss, of $\mu / r^{3}$. Bloch ${ }^{6}$ introduced the characteristic time $T_{2} \approx \hbar r^{3} / \mu^{2}$ to describe the spin-spin interaction, thus recalling the second process just mentioned. We were accustomed to use the local field as a measure of the interaction, which suggests the first process. Of course, both effects are present ordinarily and are included in any complete theory of the spin-spin interaction. $\dagger$ In any case, the examination of the line width is seen to be important in the experimental investigation of this interaction. One of the earliest results of resonance absorption experiments was the then surprising one that the line width of the proton resonance in liquids is, in general, much less than $\mu / r^{3}$ in magnitude, a result which receives quantitative explanation in the work here reported.

\footnotetext{
${ }^{6}$ F. Bloch, Phys. Rev. 70, 460 (1946).

$\dagger$ That the two effects are not one and the same can be seen by considering a lattice of non-identical nuclei, for which the second effect is absent but the "dispersion of local $z$-fields" remains.
}

The spin-spin interaction is of course incapable of bringing about heat transfer between the spin system as a whole and the thermal reservoir, or "lattice." However, the spin-spin and spinlattice interactions are not unrelated, for each involves the existence at a nucleus of perturbing fields. Therefore our attention has been directed to both effects. Broadly speaking, and anticipating results described further on, the perturbing fields responsible for spin-lattice relaxation originate in the thermal motion of neighboring magnetic dipoles, whereas the spin-spin interaction, although it may be modified by such motion, is not dependent upon it for its existence. and is in fact strongest when such motion is absent.

The picture which has been sketched in these introductory remarks will be recognized as an approximation, obtained by starting with isolated non-interacting spins, then introducing the interaction as a perturbation, but clinging still to a description in terms of individual $m_{i}$ 's. That the picture proves as useful as it does can be attributed to the fact that the spin-spin interaction is weak compared to $H_{0} \mu$ and of relatively short-range character.

Finally, we review briefly the phenomenological theory of magnetic resonance absorption, before describing the experimental method. The phenomenon lends itself to a variety of equivalent interpretations. One can begin with static nuclear paramagnetism and proceed to paramagnetic dispersion $;^{7}$ or one can follow Bloch's analysis, contained in his paper on nuclear induction, ${ }^{6}$ of the dynamics of a system of spins in an oscillating field, which includes the absorption experiments as a special case. We are interested in absorption, rather than dispersion or induction, in the presence of weak oscillating fields, the transitions induced by which can be regarded as non-adiabatic. We therefore prefer to describe the experiment in optical terms.

Consider a substance containing, per $\mathrm{cm}^{3}, N_{0}$ nuclei of spin $I$ and magnetic moment $\mu$, placed in a strong uniform magnetic field $H_{0}$ along the $z$ axis, and subjected to a weak oscillating field $I I_{x}=2 H_{1} e^{2 \pi i v t} ; H_{y}=0$. The probability of a single transition in which one of the $m_{i}$ 's changes to

${ }^{7}$ C. J. Gorter and L. J. F. Broer, Physica 9, 591 (1942). 
$m_{i}{ }^{\prime}$ can be found with the aid of the standard formula for magnetic dipole transitions:

$$
W_{m_{i} \rightarrow m_{i}{ }^{\prime}}=\left(8 \pi^{3} / 3 h^{2}\right)\left|\left(m_{i}|M| m_{i}{ }^{\prime}\right)\right|^{2} \rho_{\nu} .
$$

$M$ is the magnetic moment operator. Ordinarily $\rho_{\nu}$ represents the energy density, in unit frequency range, in the isotropic unpolarized radiation field. We have to do here with radiation of a single frequency from levels of a finite width, which we describe by the observed shape of the absorption line, $g(\nu)$. The shape function $g(\nu)$ is to be normalized so that

$$
\int_{0}^{\infty} g(\nu) d \nu=1
$$

Our radiation field is also unusual in that it consists simply of an oscillating magnetic field of one polarization. Only one of the circularly polarized components (of amplitude $H_{1}$ ) into which this field can be decomposed is effective. The equivalent isotropic unpolarized radiation density is easily arrived at, and we have for $\rho_{\nu}$

$$
\rho_{\nu}=3 H_{1}^{2} g(\nu) / 4 \pi \text {. }
$$

Introducing the nuclear gyromagnetic ratio $\gamma$, which will be used frequently hereafter,

$$
\gamma=2 \pi \mu / I h \text {, }
$$

and the matrix elements required in (1), we find

$$
\begin{aligned}
W_{m \rightarrow m-1}=(\pi / 3) \gamma^{2}(I+m)(I-m+1) \rho_{\nu} \\
=\frac{1}{4} \gamma^{2} H_{1}^{2} g(\nu)(I+m)(I-m+1) .
\end{aligned}
$$

Equation (4) gives the probability for a transition $m \rightarrow m-1$, involving the absorption from the radiation field of the energy $h \nu=h \gamma H_{0} / 2 \pi$. If the spin system is initially in equilibrium at the temperature $T$, the population of each level $m$ exceeds that of the next higher level, $m-1$, by

$$
N_{m}-N_{m-1} \approx \frac{N_{0}}{2 I+1} \frac{h \nu}{k T} \text {. }
$$

The approximation (5) is an extremely good one, for, in the cases with which we shall deal, $h \nu / k T \approx 10^{-6}$. The net rate at which energy is absorbed is now

$$
\begin{aligned}
P_{a}=\frac{N_{0}}{2 I+1} \frac{(h \nu)^{2}}{k T} \sum_{m=I}^{-I+1} W_{m \rightarrow m-1} \\
=\frac{\gamma^{2} H_{1}{ }^{2} N_{0}(h \nu)^{2} I(I+1) g(\nu)}{6 k T} .
\end{aligned}
$$

The maximum energy density in the oscillating field is $\left(2 H_{1}\right)^{2} / 8 \pi$. The apparent " $Q$ " of the sample, at frequency $\nu$, is therefore given by

$$
(1 / Q)=P_{a} / \nu H_{1}^{2}=\gamma^{2} h^{2} N_{0} I(I+1) \nu g(\nu) / 6 k T .
$$

An equivalent statement is that $\chi^{\prime \prime}$, the imaginary part of the magnetic susceptibility of the substance, is

$$
\chi^{\prime \prime}=\frac{1}{4 \pi Q}=\frac{\pi}{2}\left[\frac{N_{0} \gamma^{2} \hbar^{2} I(I+1)}{3 k T}\right] \nu g(\nu),
$$

where $\hbar=h / 2 \pi$. The term in brackets will be recognized as the static susceptibility $\chi_{0}{ }^{\prime}$ of the spin system. It may be noted in passing that Eq. (8) is compatible with, and could have been obtained from, the Kramers formula connecting the real and imaginary parts of the susceptibility, which for this special case is

$$
\chi_{0}{ }^{\prime}=\frac{2}{\pi} \int_{0}^{\infty} \frac{\chi^{\prime \prime}(\nu) d \nu}{\nu} .
$$

If our earlier estimate of the line width is correct, the maximum value of $g(\nu)$ should be of the order of magnitude $h r^{3} / \mu^{2}$; if so, the maximum value of $\chi^{\prime \prime}$ to be expected, according to Eq. (8), is of the order of $h \nu / k T$, since $N_{0} \approx 1 / r^{3}$. A more accurate estimate based on the theory to be given later leads to the formula:

$$
\chi^{\prime \prime}{ }_{\max } \approx 0.2(h \nu / k T)[I(I+1)]^{\frac{1}{2}} .
$$

The numerical factor in (10) depends somewhat on the geometrical arrangement of the nuclei. For $\nu=10 \mathrm{Mc} / \mathrm{sec}$., and room temperature, $h \nu / k T=1.64 \times 10^{-6}$. If the line is much narrower than the above estimate-as we shall find it to be in many cases-the maximum value of $\chi^{\prime \prime}$ is correspondingly larger. The experimental detection of the absorption thus presents the problem of detecting a small change in the susceptibility of the core of a radiofrequency coil. The absorption is, of course, accompanied by dispersion, the maximum value of $\chi^{\prime}$ being of the same order of magnitude as the maximum value of $\chi^{\prime \prime}$. The dispersive properties of the medium, moreover, are those of a substance displaying the Faraday effect, except that the effect is greatly enhanced by resonance. Ad- 
vantage is taken of this in the nuclear induction method, but not in our experiments.

The formula (8) applies regardless of the origin of the line broadening, even if the line width and shape are controlled, as is often the case, by accidental inhomogeneities in the supposedly uniform $H_{0}$, provided that the original distribution of population among the levels remains substantially unaltered. In other words (8) is correct for vanishing $H_{1}$. As $H_{1}$ is increased, the thermal contact between spin system and lattice eventually proves unable to cope with the energy absorbed by the spin system, the spin temperature rises, and the relative absorption, measured by $\chi^{\prime \prime}$, diminishes. It is the onset of this saturation effect which has been used to measure the spin-lattice relaxation time in most of our work. The interpretation of the data is complicated by the necessity of distinguishing various cases according to the factors which control the line width, and a detailed discussion of this problem will be postponed. However, in order to introduce at an early stage our working definition of the spin-lattice relaxation time, we shall now investigate the occurrence of saturation in a simple case.

Assume that $I=\frac{1}{2}$, so that we have to deal with two levels. Let $n$ denote the surplus population of the lower level: $n=N_{+\frac{1}{1}}-N_{-\frac{1}{1}}$, and let $n_{0}$ be the value of $n$ corresponding to thermal equilibrium at the lattice temperature. We assume that, in the absence of the radiofrequency field, the tendency of the spin system to come to thermal equilibrium with its surroundings is described by an equation of the form

$$
d n / d t=\left(1 / T_{1}\right)\left(n_{0}-n\right) .
$$

The assumption will be justified in Section VII. The characteristic time $T_{1}$ in Eq. (11) is called the spin-lattice relaxation time. (Wherever possible our definitions and nomenclature follow those of Bloch.) ${ }^{6}$

The presence of the radiation field requires the addition to (11) of another term :

$$
d n / d t=\left(1 / T_{1}\right)\left(n_{0}-n\right)-2 n W_{3 \rightarrow-3} .
$$

A steady state is reached when $d n / d t=0$, or, using Eq. (4), when

$$
n / n_{0}=\left[1+\frac{1}{2} \gamma^{2} H_{1}^{2} T_{1} g(\nu)\right]^{-1} .
$$

We shall express the maximum value of $g(\nu)$ in terms of a quantity $T_{2}{ }^{*}$ defined by

$$
T_{2}{ }^{*}=\frac{1}{2} g(\nu)_{\max } \text {. }
$$

The maximum steady-state susceptibility in the presence of the $r-f$ field is thus reduced, relative to its normal value, by the "saturation factor" $\left[1+\gamma^{2} H_{1}{ }^{2} T_{1} T_{2}{ }^{*}\right]^{-1}$. The possibility that $T_{2}{ }^{*}$ itself depends on $H_{1}$ is not excluded.

The quantity $T_{2}{ }^{*}$ defined by (14) is a measure of the inverse line width. It is strictly connected only with the area and peak value of a line-shape curve. In the case of the damped oscillator or single-tuned-circuit curve, $1 / \pi T_{2}{ }^{*}$ is the full width between the half-maximum points, on a frequency scale. We use the notation $T_{2}{ }^{*}$ when admitting all sources of line broadening, including inhomogeneity in the magnetic field. The symbol $T_{2}$ will be used to specify the true line width which is observable only if the field is sufficiently homogeneous.

\section{APPARATUS AND EXPERIMENTAL PROCEDURE}

The substance to be investigated was located within a small coil, in the field of an electromagnet. Resonance absorption in the sample caused a change in the balance of a radiofrequency bridge containing the coil. In much of the work a frequency in the neighborhood of $30 \mathrm{Mc} / \mathrm{sec}$. was used. This is a convenient region in which to study the $\mathrm{H}^{1}$ and $\mathrm{F}^{19}$ resonances which occur at $30 \mathrm{Mc} / \mathrm{sec}$. in fields of 7050 and 7487 gauss, respectively. Some experiments were made at $14.5 \mathrm{Mc} / \mathrm{sec}$. and at 4.5 $\mathrm{Mc} / \mathrm{sec}$. Considerations of sensitivity alone favor the highest frequency compatible with available magnetic field intensities and the gyromagnetic ratio of the nucleus in question. (See Appendix.)

A typical $r-f$ coil consists of 12 turns of No. 18 copper wire wound in a helix of inside diameter $7 \mathrm{~mm}$, and $15 \mathrm{~mm}$ long. The coil is sometimes supported on a thin-walled polystyrene form, but this is not always desirable for, in addition to reducing the space available for the sample, the coil form, if it contains hydrogen, gives rise to an absorption at the proton resonance. $\dagger$ The

t† An excellent insulating material of negligible hydrogen content is Teflon, the Dupont fluorine-substituted polymer. We have not been able to detect a proton resonance in Teflon. Its mechanical properties, however, are not ideal for a coil form. Glass would be acceptable, as would porcelain. 
coil is surrounded by a cylindrical shield of 17 $\mathrm{mm}$ inside diameter, one end of the coil being connected to the shield. A hole in the end of the shield can permits insertion of the sample which, if a liquid or powder, is contained in a thin-walled glass tube. The effective volume of the sample within the coil is approximately $0.5 \mathrm{~cm}^{3}$. It would be advantageous to work with larger samples; in the present case the limitation is imposed by the width of the magnet gap. For experiments requiring lowering of the temperature, copper tubing was wound around and soldered to the shield can, and acetone cooled by dry ice was allowed to flow through the cooling tube at a controllable rate. This rather clumsy arrangement was also necessitated by lack of space in the magnet gap.

The magnet is a Sociéte Genevoise watercooled electromagnet provided with pole pieces 4 inches in diameter at the gap. The gap width was adjustable and was ordinarily between $\frac{3}{4}$ and 1 inch. The pole faces were flat except for a raised rim 0.015 inch high and 0.31 inch wide at the periphery of each, which was designed according to the prescription of $\operatorname{Rose}^{8}$ to compensate the inherent radial inhomogeneity of the simple gap. The residual inhomogeneities were apparently of non-geometrical origin, and doubtless arose from magnetic inhomogeneities in the cold-rolled steel pole caps. More careful attention to the pole cap material, in future work, is to be recommended. The best position in the field was found by exploring with a liquid sample. As will be seen later, the line width in water, for example, is essentially determined by the field inhomogeneity, and therefore affords a sensitive test for homogeneity. The location of the flattest spot in the field varied somewhat with field intensity, which is evidence for ferromagnetic inhomogeneity of the poles. The magnet current was supplied by storage batteries and regulated by suitable rheostats, the main rheostat consisting of some 15 feet of heavy Advance strip, with a sliding contact. It will be readily appreciated that the examination of resonance lines a few tenths of a gauss in width in fields of several thousand gauss requires an unusually stable source of current. The current could be monitored by means of a shunt and a type $\mathrm{K}$ potentiometer. The power required for magnet excitation at 7000 gauss with a 0.75 -inch gap was about 220 watts.

A sinusoidal modulation of small amplitude, at 30 c.p.s., was applied to the magnetic field by means of auxiliary coils around the poles, or, in some cases, by means of a small pair of coils

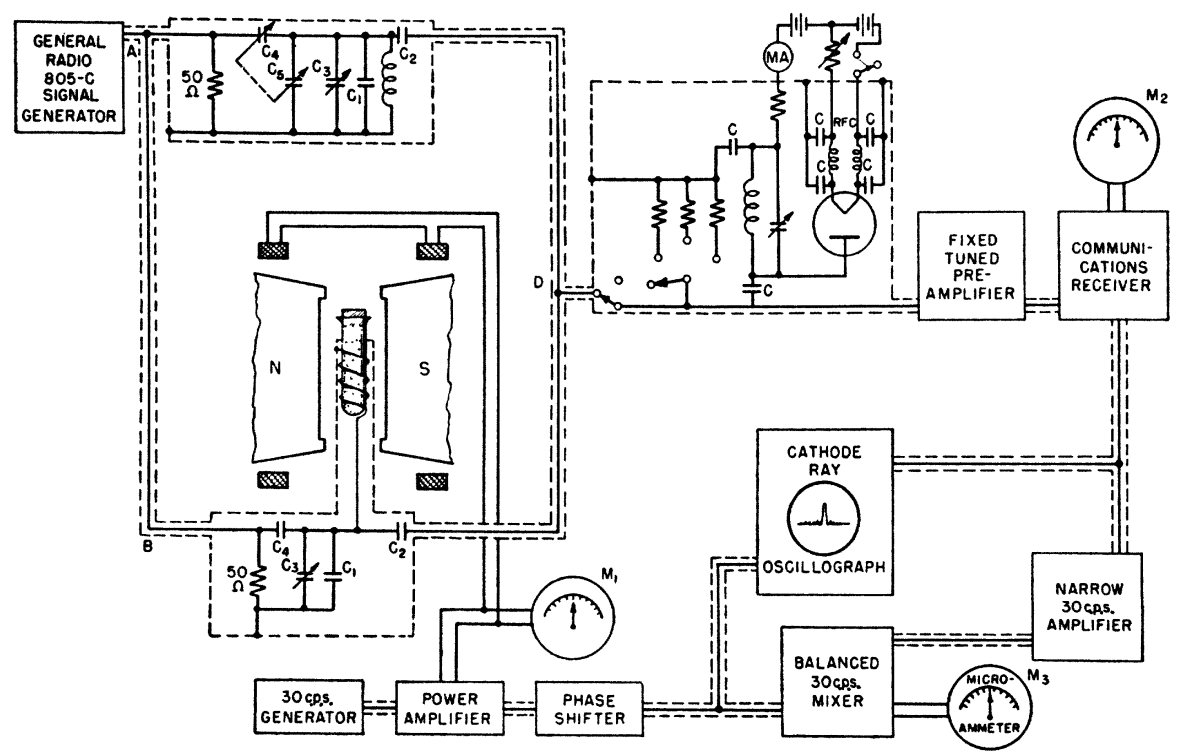

FIG. 1. Block diagram of circuit. The sample and the magnet gap are not drawn in correct proportion.

${ }^{8}$ M. E. Rose, Phys. Rev. 53, 715 (1938). 
fitting closely against the shield surrounding the r-f coil. The 30-c.p.s. signal was derived from a small permanent-magnet alternator driven by a synchronous motor, and a 6L6 served as power amplifier to drive the modulating coils. The amplitude of modulation at the sample could be made as large as $\mathbf{1 5}$ gauss peak-to-peak, but was usually very much smaller.

A block diagram of the radiofrequency circuit is shown in Fig. 1. The source of the r-f signal was a General Radio 805-C signal generator. The output of this instrument covers a convenient range in power, the maximum power available being adequate to saturate most samples. The circuit elements are connected together by standard u.h.f. coaxial cable (50 ohms). Each of the two branches leading from a tee-junction near the signal generator contains a resonant circuit, one of which consists of the coil surrounding the sample and a fixed ceramic condenser shunted by a variable condenser of about $10 \mathrm{mmf}$. The dummy circuit in the other branch is similar in all respects but is not located in the field. When liquids of high dielectric constant are being investigated it is sometimes worth while to slip a dummy sample into the dummy coil, thus balancing, at least approximately, the considerable change in stray capacitance resulting from the insertion of the sample in the other coil.

An extra half-wave-length of line $A B$ in one branch makes the point $D$, where the branches join to the line to the amplifier, a voltage node in the perfectly balanced condition. This is, of course, only one of many ways in which the equivalent of a balanced-pair to unbalanced line transition, essential to any bridge, might be effected, and simplicity is the only virtue claimed for it. The $Q$ of a coil such as that described is about 150 , at $30 \mathrm{Mc} / \mathrm{sec}$, and the shunt impedance of the $L C$ circuits at resonance is of the order of $5000 \mathrm{ohms}$. The small coupling condensers $C_{2}$ are chosen to make the impedance seen looking back from the amplifier approximate the value for which the amplifier noise figure is best. The main tuning capacity in each circuit is provided by a fixed ceramic condenser $C_{1}$.

The bridge is balanced in phase and amplitude by the adjustment of one of the trimming tuning condensers $C_{3}$ and the coupling condenser $C_{4}$.
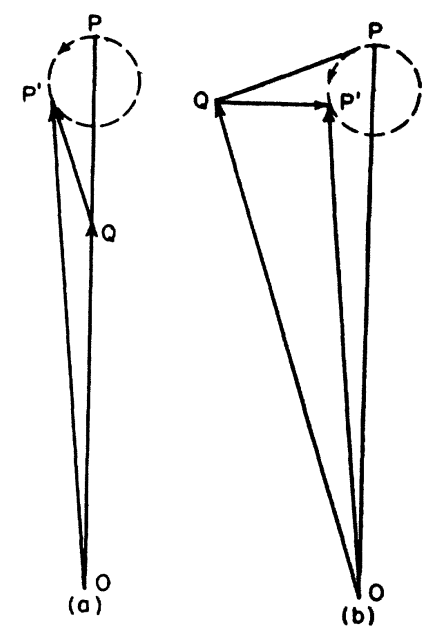

FIG. 2. Diagram illustrating the dependence of the shape of the resonance curve upon the nature of the residual unbalance of the bridge. The diagram is not to scale; actually, $P P^{\prime} \ll Q P \ll O P$.

The latter adjustment alone affects the phase balance as well as the amplitude balance, for the coupling condenser essentially appears in shunt with the tuned circuit. The adjustable coupling condenser $C_{4}$ therefore is ganged with a trimming tuning condenser $C_{5}$ in such a way as to leave the total tuning capacity unaltered during the change in coupling. In this way substantially orthogonal phase and amplitude adjustments are provided, and the balancing of the bridge is a simple operation.

In searching for, or examining, a nuclear resonance the bridge is never completely balanced, but is intentionally unbalanced to such a degree that the voltage appearing at point $D$ is large compared to the change in this voltage caused by nuclear absorption or dispersion. The only benefits derived from the use of the bridge are (a) the reduction of the $r-f$ level at the input to the amplifier to a value low enough to permit considerable $\mathrm{r}-\mathrm{f}$ amplification before detection, and (b) the reduction in the relative magnitude of output fluctuations arising from amplitude fluctuations in the signal supplied by the signal generator. With a rather moderate degree of balance these advantages are already fully exploited, and further balance does not improve matters. We have ordinarily operated with a balance of $40 \mathrm{db}$ to $60 \mathrm{db}$, by which is meant that the voltage appearing at $D$ is between $10^{-2}$ 


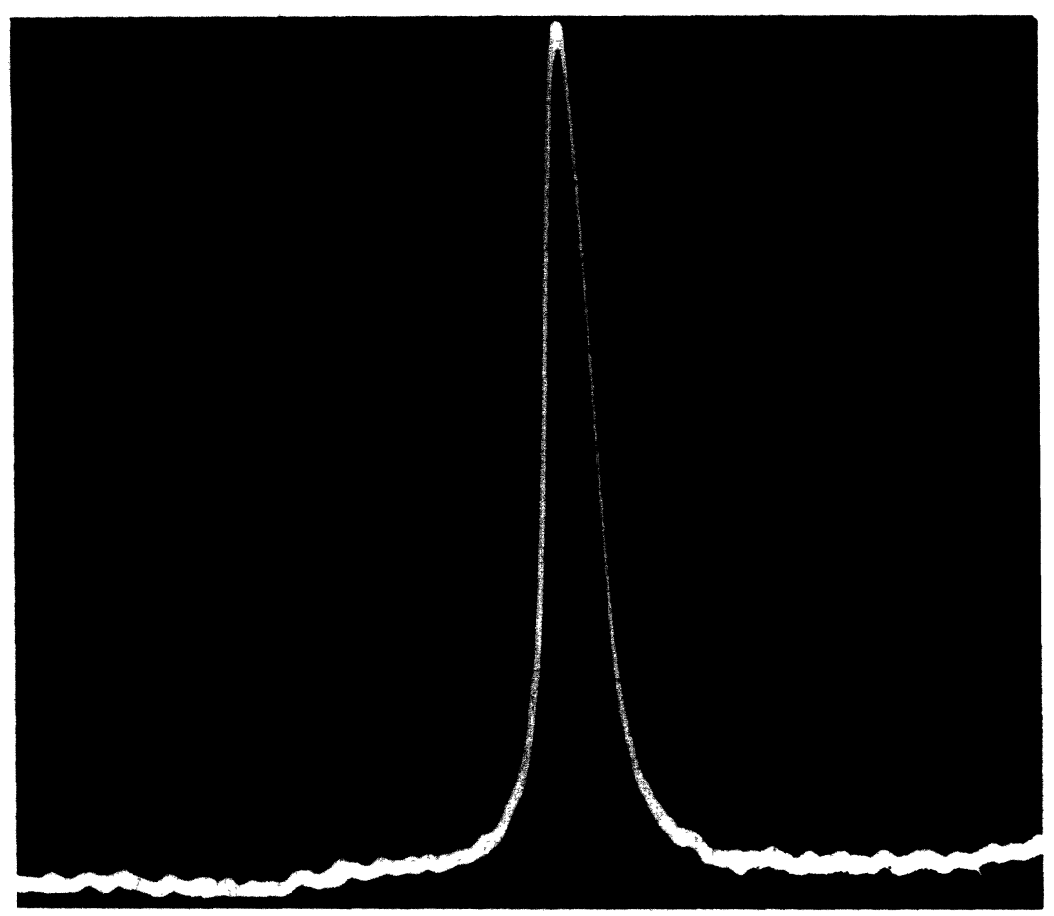

Fig. 3. Proton resonance (absorption curve) in ferric nitrate solution. and $10^{-3}$ times the value it would have if one branch were removed.

It is important to be able to control the character of the residual unbalance, for upon this depends the nature of the signal observed as the magnetic field is varied through the region of resonance. The nuclear absorption has the effect of altering the shunt resistance of the tuned circuit, while the accompanying nuclear dispersion changes its resonant frequency slightly. The two effects can be described together by considering, in Fig. 2, the vector $O P^{\prime}$ as representing the signal arriving at the point $D$ from the branch containing the $L C$ circuit with the sample in it. As resonance is traversed the end of the vector, $P^{\prime}$, describes a small circle. t† $^{\prime}$ Had the residual unbalance off resonance, $Q P$, referred to the point $D$, been in amplitude only, so that the signal from the other branch is represented by the vector $O P$ in Fig. 2, the resultant voltage applied to the amplifier, $Q P^{\prime}$, would vary with magnetic field in the manner illustrated in Fig. 3 which is a trace of the receiver output after detection, vs. magnetic

\footnotetext{
tt† The locus of $P^{\prime}$ is strictly circular only if the shape of the absorption curve is that appropriate to a damped oscillator.
}

field, in the case of a proton resonance. Pure phase unbalance, on the other hand, is represented by the diagram of Fig. $2 b$, and the observed output variation is shown in Fig. 4. (It must be remembered that actually the resultant $Q P^{\prime}$ is very much smaller than $O P$, and the small circle is in turn very much smaller than the unbalance voltage $Q P$.) It is, in fact, the nuclear absorption, $\chi^{\prime \prime}$, which is observed in Fig. 3 and the nuclear dispersion, $\chi^{\prime}$, which is observed in Fig. 4. Either can be obtained as desired by first balancing the bridge to a high degree and then setting in an intentional unbalance by means of the phase knob or the amplitude knob-hence the importance of independent phase and amplitude controls. A "mixed" unbalance brings about an unwelcome mixture of the two effects recognizable by the unsymmetrical shape of the output curve. We usually preferred to work with the absorption curve (amplitude unbalance) because the apparatus is in this condition less susceptible to the frequency modulation present in the signal generator output. Indeed, absence of frequency modulation effects in the output is a sensitive criterion for pure amplitude unbalance. The trouble caused by frequency modulation is, of course, mainly due 
to the frequency sensitivity of the half-wave line, and could be mitigated by the use of a suitably designed transformer to achieve the required $180^{\circ}$ phase shift.

The $r-f$ amplifier is subject to no special requirements other than the desirability of a good noise figure. We have used commercial receivers, both Hallicrafters S-36 and National HRO, preceded, when the signal frequency is near $30 \mathrm{Mc} / \mathrm{sec}$., by a broad band preamplifier of Radiation Laboratory design ${ }^{9}$ which has a much smaller noise figure than either of the commercial receivers. A temperature-limited diode in a circuit for noise comparison (shown in Fig. 1) has been included as a permanent fixture, and calibration by this means showed the noise figure to be $1.5 \mathrm{db}$ when the preamplifier was in use.

Relatively strong signals, such as that displayed in Fig. 3, can be observed on an oscilloscope by merely connecting the detector output to the vertical deflection amplifier, and synchronizing the sweep with the 30 -c.p.s. modulation of the magnetic field, the amplitude of which is adjusted to several times the line width. For most of our work, however, we have used, following the $r-f$ amplifier and detector, a narrow band amplifier and balanced mixer, a combination variously known as a "lock-in" amplifier or a phase-sensitive detector. The magnetic field modulation is reduced to a fraction of the line width, and the resulting 30-c.p.s. modulation of the detected receiver output is amplified and converted by mixing it with a 30 -c.p.s. reference signal derived from the small alternator. There is thus obtained a d.c. output proportional to the slope of the nuclear absorption curve, and this is indicated by a meter $M_{3}$. The relation between meter current and $d \chi^{\prime \prime} / d H$ is strictly linear for sufficiently small amplitudes of magnetic field modulation, because of the relatively large unbalance voltage in the bridge and the nature of the lock-in amplifier. The effective band width of the system is determined by the time constant of the final d.c. circuit containing the meter, which was usually about 1 second but could be increased to 10 seconds if desired. The amplifier was copied after one designed by R. H. Dicke. ${ }^{10}$

The superiority of the narrow-band-amplifierplus-meter over the oscilloscope in the matter of detecting weak signals is not as marked as

FIG. 4. Proton resonance (dispersion curve) in ferric nitrate solution

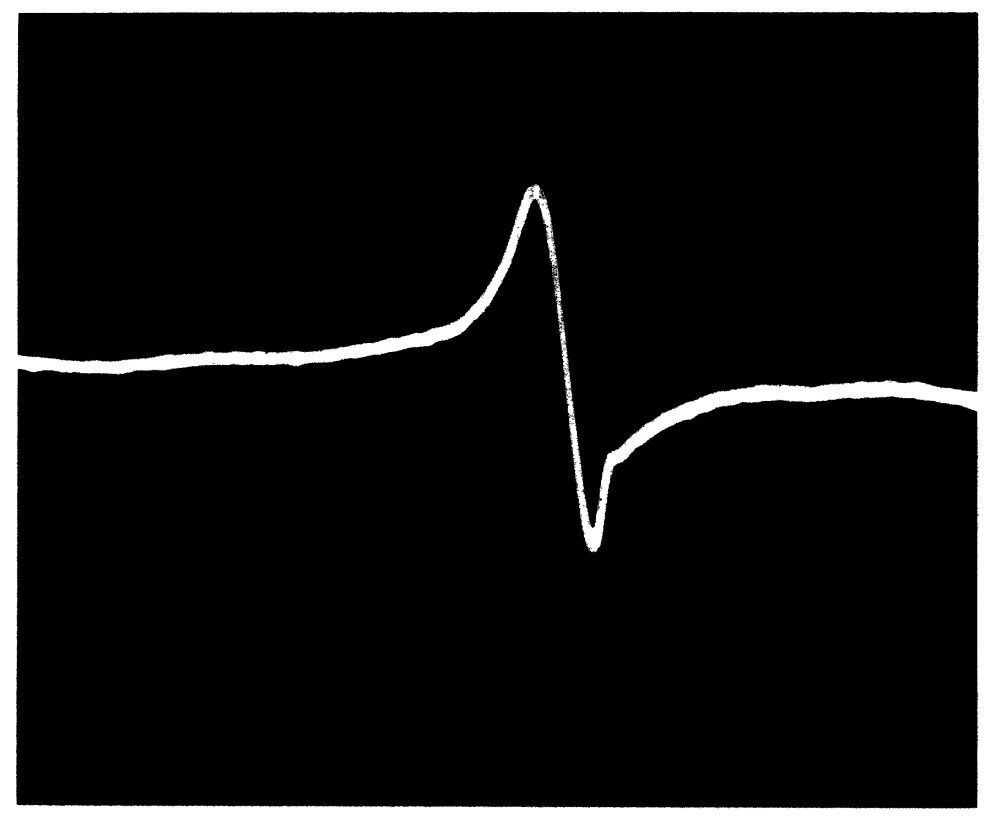

${ }^{\circ}$ G. E. Valley and H. Wallman, Vacuum Tube Amplifiers, Radiation Laboratory Series (McGraw-Hill Book Company, Inc., New York, in press), Vol. 18.

${ }^{10}$ R. H. Dicke, Rev. Sci. Inst. 17, 268 (1946). 
comparison of the band widths alone would suggest, because of the new well recognized ability of the observer to integrate noise. However, for the quantitative examination of weak lines the narrow-band device is nearly indispensable.

It will be noted that the output of the lock-in amplifier, in the case of amplitude unbalance, reproduces the derivative of the absorption curve (which resembles qualitatively a dispersion curve), while with phase unbalance one obtains the derivative of the dispersion curve - a large peak flanked symmetrically by two dips.

\section{MEASUREMENT OF LINE WIDTH}

When the resonance absorption is intense enough to be displayed on the oscilloscope screen the line width can be measured directly on the trace. A sinusoidal horizontal sweep synchronous with, and properly phased with respect to, the modulation of the magnetic field, provides a horizontal scale linear in gauss. We denote by $H_{m}$ the modulation amplitude, and by $\omega_{m}$ the angular frequency of modulation, in these experiments $2 \pi \times 30 \mathrm{sec}^{-1}$. That is, $H_{z}=H_{0}+H_{m} \sin \omega_{m} t$. To establish the scale against which the line width is measured, $H_{m}$ must be known. This calibration can be effected in several ways. The signal generator frequency can be altered by a small, known amount, and the resulting shift of the resonance within the modulation interval noted, or the alternating voltage induced in a small coil of known turns $\times$ area, at the location of the sample, can be measured with a vacuumtube voltmeter. Both methods were used and gave consistent results. The relation between $H_{m}$ and the voltage applied to the modulating coils was so nearly linear that a single calibration usually sufficed for a given setting of the gap width.

The band width of the system must, of course, be adequate to permit a faithful reproduction of the line shape at the modulation frequency used. The $r-f$ field applied to the sample must be weak enough to avoid saturation. If the line is very narrow a complication can arise from a transient effect to be described briefly later. The vestige of such an effect is responsible for the asymmetry at the base of the absorption line in Fig. 3 .

For the study of broad, and hence weak, absorption lines the narrow-band amplifier is used. The modulation amplitude $H_{m}$ is reduced to a fairly small fraction of the line width, and the line is explored by slowly changing the magnetic field $H_{0}$. It usually suffices to note the interval in $H_{0}$ between points giving extreme deflection of the output meter $M_{3}$, which are assumed to be the points of maximum slope of the absorption curve. A plot of the output meter readings in one case is shown in Fig. 5. In this example, the interval between extreme deflections is $\Delta H=0.5$ gauss (curve $a$ ). The relation between $\Delta H$ so determined and $T_{2}$ defined by Eq. (14) would be $\left(1 / T_{2}\right)=(\sqrt{3} / 2) \gamma \Delta H$, for a damped oscillator absorption curve, or $\left(1 / T_{2}\right)$ $=(2 \pi)^{-\frac{1}{3}} \gamma \Delta H$, for a Gaussian curve. The derivative curve $(a)$ in Fig. 5 indicates an absorption line shape of intermediate character. The most conspicuous evidence for this is the ratio of the maximum negative to the maximum positive

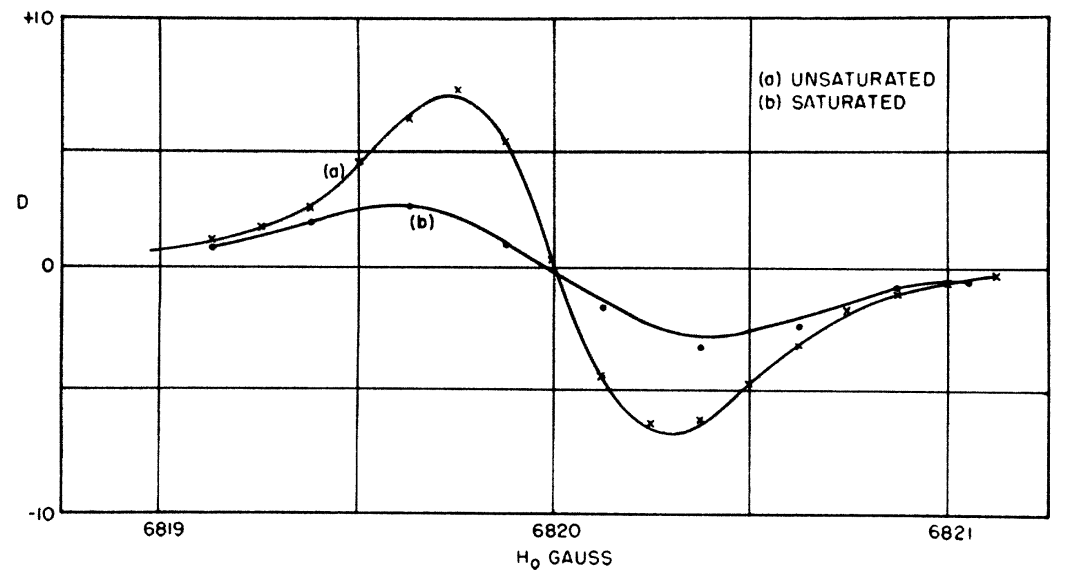

FIG. 5. Plot of the reading of the output meter $M_{3}$, for a $0.5 \mathrm{~N}$ solution of ferric nitrate. Curve (b) was taken at a high power level and shows the effect of saturation. The value of the magnetic field at the line center was not measured, but merely computed from the frequency and the accepted value of the proton moment. 
slope, which should be $4: 1$ for the derivative of a damped oscillator curve, 2.2:1 for the derivative of a Gaussian, and is in fact about $3: 1$.

The effect of saturation, evident in the curve (b) in Fig. 5, is to flatten and spread apart the extrema of the derivative curve. This comes about because saturation is more nearly complete at the center of the line.

\section{MEASUREMENT OF RELAXATION TIME}

Two methods by which the spin-lattice relaxation time $T_{1}$, defined by Eq. (11), can be measured have been mentioned in the introduction. The direct observation of recovery from saturation was convenient only when $T_{1}$ was of the order of magnitude of one second; doubtless a wider range could be covered with suitable circuit modifications and recording instruments. The direct method has the advantage that the interpretation of the results is simple and entirely unambiguous. The saturation-curve method is applicable over a much wider range, but the analysis of the experiment is rather involved and absolute values of $T_{1}$ derived in this way are probably less reliable. The two methods complement one another, as we shall see.

Before analyzing special cases, we shall describe an instructive experiment which vividly demonstrates the saturation phenomenon and also exposes the source of some of the complications which are encountered in applying the saturation-curve method to narrow resonance lines.

The proton resonance in water was observed on the oscilloscope with a weak (non-saturating) $\mathrm{r}-\mathrm{f}$ field. The line appeared as in Fig. 6(a). The modulation amplitude $H_{m}$ was then reduced to zero for a few seconds, while the field $H_{0}$ was maintained at a value corresponding to the point $A$; the line was, of course, not visible on the screen during this interval. Upon turning the modulation amplitude back to its original value, and restoring the sweep, the line reappeared bearing a deep, narrow "gash" at the position $A$, as in Fig. 6(b). This hole rapidly filled up and in a few seconds was gone, as shown in the sketches (c) to (e). The explanation of the effect is this: the true width of the water resonance line was much less than the width of the original curve, which was caused entirely by
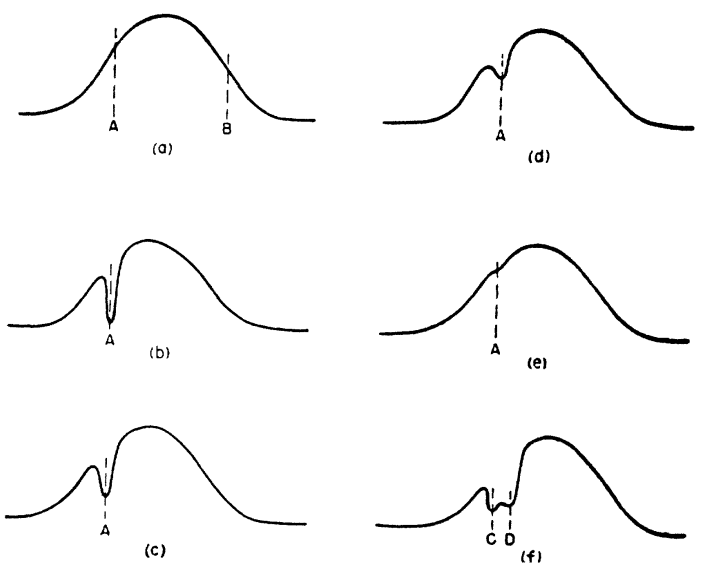

FIG. 6. The effect of local saturation upon a line whose width is caused by magnetic field inhomogeneity.

inhomogeneity of the field $H_{0}$. The absorption observed at $A$ was caused by nuclei in one small part of the sample, that at $B$ by those in another part. With the removal of the modulation the former group was subjected to radiation at its resonance frequency all the time, and was more or less thoroughly saturated. The subsequent recovery from saturation occurred when the modulation was restored. The gash in the curve betrays, quite literally, a hot spot (in respect to nuclear spin temperature) somewhere in the sample.

If the modulation amplitude $H_{m}$ is reduced to a small but finite value, rather than zero, the r-f amplitude $H_{1}$ being meanwhile increased somewhat, subsequent observation will disclose a hole like that in Fig. 6(f), which then heals at the same rate as before. The double dip results, of course, from the sinusoidal modulation-a longer time was spent at the turning points $C$ and $D$ than at the middle of the hole. $\neq$

If the attempt is made to produce the effect shown in Fig. 6 in a substance for which the line width is determined by a strong spin-spin interaction, rather than field inhomogeneity, it is found that the whole curve saturates; it is not possible to "eat a hole" in the curve. The energy

$\ddagger$ It was the observation of the effects just described which first made us aware that the true line width in water was much less than the apparent line width. By exploring the magnet gap, a spot was found where the field was considerably more homogeneous and the line correspondingly narrower. The narrowest line obtainable in our magnet at 7000 gauss was about 0.1 gauss wide; at 1100 gauss a width of 0.015 gauss was achieved in one case. 
absorbed by the spins can no longer be localized, but goes to raise the temperature of the spin system as a whole. In other words, the application of an r-f field at a single frequency, with $H_{0}$ fixed, immediately (or at least in a time shorter than $T_{1}$ ) affects a region about $2 / \gamma T_{2}$ wide on a magnetic field scale, or about $1 / \pi T_{2}$ wide on a frequency scale. It is important to keep this point in mind.

In any case, it is possible to saturate all parts of the sample by modulating through the whole line, using the highest available $r$-f field intensity. The recovery, observed with a much weaker $r-f$ field in the sample, appears to follow the simple law $\left(1-e^{-t / t_{0}}\right)$, and we identify $t_{0}$ with $T_{1}$, the spin-lattice relaxation time. To make a quantitative measurement, the oscilloscope trace was photographed with a movie camera operating at a known frame speed, and the film analyzed to determine the time constant of recovery. This is the direct method referred to above. It was applied to a distilled water sample, to petroleum ether, and to a $0.002 \mathrm{~N}$ solution of $\mathrm{CuSO}_{4}$, yielding relaxation times of $2.3 \pm 0.5 \mathrm{sec}$., 3.0 \pm 0.5 sec., and $0.75 \pm 0.2 \mathrm{sec}$., respectively.

The saturation curve method is based upon Eq. (13) in the Introduction. If it were possible to measure, in the steady state, the value of $\chi^{\prime \prime}{ }_{\max }$ which is reduced from its normal unsaturated value by the factor $\left(1+\gamma^{2} H_{1}^{2} T_{1} T_{2}\right)^{-1}$, a knowledge of $H_{1}$ and $T_{2}$ would then suffice to determine $T_{1}$. In the modulation method, however, we measure $\partial \chi^{\prime \prime} / \partial H_{0}$, which is equivalent to measuring $\partial \chi^{\prime \prime} / \partial \omega$. This introduces some complications which can be treated briefly only by confining the discussion to typical limiting cases.

We assume for analytical convenience that the shape of the unsaturated absorption curve is that appropriate for a damped oscillator,

$$
g(\nu)=\frac{2 T_{2}}{1+4 \pi^{2}\left(\nu-\nu_{0}\right)^{2} T_{2}^{2}}=\frac{2 T_{2}}{1+\left(\omega-\omega_{0}\right)^{2} T_{2}^{2}} .
$$

In Eq. (15), $\omega_{0}$, the center angular frequency, is $\gamma H_{0}{ }^{*}$. Although it is actually $H_{z}$ which is varied in the course of the experiment, both by applying the modulation $H_{m} \sin \omega_{m} t$, and by slowly changing the magnet current in order to move through the resonance curve, it is convenient here to think of $H_{0}$ as fixed at the central value $H_{0}{ }^{*}$, with $\omega$ as the variable. The modulation $H_{m}$ $\sin \omega_{m} t$ is equivalent to a frequency modulation of amplitude $\gamma H_{m}$ radians/sec. If the amplitude of the modulation is small enough, $\gamma H_{m}$ $<1 / T_{2}$, the deflection of the output meter $M_{3}$ will be nearly proportional to $\partial \chi^{\prime \prime} / \partial H_{0}$, or, in terms of the equivalent frequency modulation, to $\partial \chi^{\prime \prime} / \partial \omega$. The question is, what is to be regarded as constant in interpreting $\partial \chi^{\prime \prime} / \partial \omega$ in the presence of saturation.

We first consider cases in which the inhomogeneity of the magnetic field, denoted roughly by $\delta H$, has a negligible influence on the line width. That is, $\gamma \delta H \ll 1 / T_{2}$. The apparent susceptibility of the sample in the steady state (no modulation) at the frequency $\omega_{1}$, relative to its unsaturated value at $\omega_{0}$, the line center, is

$$
\begin{aligned}
& \chi^{\prime \prime}\left(\omega_{1}, H_{1}\right) / \chi^{\prime \prime}\left(\omega_{0}, 0\right) \\
& \quad=\left(1+x^{2}\right)^{-1}\left[1+s /\left(1+x^{2}\right)\right]^{-1}=f(x) S(x),
\end{aligned}
$$

evaluated at $x=x_{1}$, were $x=\left(\omega-\omega_{0}\right) T_{2}$ and $s=\gamma^{2} H_{1}^{2} T_{1} T_{2}$. The factor $\left[1+s /\left(1+x^{2}\right)\right]^{-1}=S(x)$ is the saturation factor given by Eq. (13). We are interested in $\partial \chi^{\prime \prime} / \partial x$, and, in particular, we ask for the extreme values which the derivative attains as $x_{1}$ is varied. These will be assumed proportional to the extreme meter deflections, $D_{\text {ex }}$, indicated on Fig. 5. There are two cases to be distinguished according to the relative magnitude of $\omega_{m}$ and $1 / T_{1}$. If $\omega_{m} T_{1} \ll 1$, the saturation factor $S(x)$ will vary during a modulation cycle. If $\omega_{m} T_{1} \gg 1, S$ cannot change during the modulation cycle and may be assigned a value appropriate to the center of the modulation swing. The meter deflection $D$ is therefore proportional, in the two cases, to different functions of $x_{1}$, namely:

Case 1

$$
\left(\omega_{m} T_{1} \ll 1\right),\left.\quad D\left(x_{1}\right) \propto \frac{\partial}{\partial x}[f(x) S(x)]\right|_{x=x_{1}} ;
$$

Case 2

$$
\left(\omega_{m} T_{1} \gg 1\right),\left.\quad D\left(x_{1}\right) \propto S\left(x_{1}\right) \frac{\partial}{\partial x} f(x)\right|_{x=x_{1}} .
$$

$D_{\text {ex }}$, the extreme excursion of $D$, is found by an elementary computation to depend in the follow- 
ing way upon the saturation parameter $s$ :

Case 1

Case 2

$$
D_{\mathrm{ex}} \propto(1+s)^{-\frac{3}{2}}
$$

$$
\begin{aligned}
& D_{\text {ex }} \propto 8\left[2\left(16+16 s+s^{2}\right)^{\frac{1}{2}}-2 s-4\right]^{\frac{1}{3}} \\
& \quad \times\left[8+8 s-s^{2}+(2+s)\left(16+16 s+s^{2}\right)^{\frac{1}{2}}\right]^{-1} .
\end{aligned}
$$

The functions (17) and (18) are plotted in Fig. 7. The saturation effect makes itself evident more rapidly in Case 1, as was to be expected. A different line shape would, of course, lead to somewhat different results.

Suppose now that the field is not homogeneous, and, in particular, that $H_{m}<1 / \gamma T_{2}<\delta H$, which we shall label Case 3. The result is then simply a superposition, governed by $\delta H$, of curves of the sort analyzed in either Case 1 or Case 2 (depending on the magnitude of $\omega_{m} T_{1}$ ). If the line shape caused by the field inhomogeneity were known, the appropriately weighted superposition of curves $D\left(x_{1}\right)$ could be constructed. It is clear, however, that the decrease in $D_{\text {ex }}$ with $s$ will follow much the same course; $D_{\text {ex }}$ will be substantially reduced when $s$ is of the order of unity.

Case 4 , specified by $1 / \gamma T_{2}<H_{m}<\delta H$, and $\omega_{m} T_{1}>1$, is significantly different. It is now the modulation amplitude $H_{m}$ rather than $1 / \gamma T_{2}$, which determines the width of the region momentarily saturated. The situation is, in fact, that illustrated by Fig. 6(f). An analysis of this case, which is only feasible if certain simplifying assumptions are made, shows that the quantity $\gamma H_{1}^{2} T_{1} / H_{m}$, rather than $\gamma^{2} H_{1}^{2} T_{1} T_{2}$, plays the role of the saturation parameter, a rapid decrease in $D_{\text {ex }}$ occurring when $\left(\gamma H_{1}^{2} T_{1} / H_{m}\right) \approx 1$. The indicated dependence of $H_{1}$ upon $H_{m}$, for the same degree of saturation, has been confirmed experimentally. Case 4 is encountered in liquids with relatively long relaxation times. The measurements of $D_{\text {ex }}$ are particularly difficult here for the line must be traversed at an extremely slow rate in order to permit a quasi-stationary state to develop at every value of $x_{1}$.

An even more complicated case is defined by $1 / \gamma T_{2}<H_{m}<\delta H$ with $\omega_{m} T_{1}<1$. The discussion of this case will be omitted as it was not an important one experimentally.

An experimental saturation curve is obtained by the following procedure. The modulation amplitude, $H_{m}$, is set at a suitable value and remains the same throughout a run. The output voltage of the signal generator, to which $H_{1}$ is proportional, is varied in steps by means of the attenuator in the instrument. At each step the receiver gain is adjusted to bring the rectified $r-f$ output of the receiver to a fiducial level as indicated by the voltmeter $M_{2}$, the bridge balance remaining unaltered. This makes the constant of proportionality connecting the deflection of the final output meter with $\partial \chi^{\prime \prime} / \partial H_{0}$ the same for each step in $H_{1}$. At each signal generator output setting, after the receiver gain has been readjusted, the resonance curve is slowly traversed and the maximum and minimum readings of the output meter noted. The difference between the readings is then plotted against the logarithm of the signal generator voltage.

To interpret the saturation curves we assume that a given degree of saturation is associated with the same value of $\gamma^{2} H_{1}^{2} T_{1} T_{2}$, or, if the conditions correspond to Case 4, of $\gamma H_{1}^{2} T_{1} / H_{m}$. This is, of course, not strictly correct if we are comparing two saturation curves which correspond to two different cases, such as Case 1 and Case 2, and allowance can be made if the precision of the data warrants.

A series of saturation curves for $\mathrm{Fe}\left(\mathrm{NO}_{3}\right)_{3}$ solutions of various concentrations is shown in Fig. 8. This is the roughest set of curves of any used in deriving the results reported in this

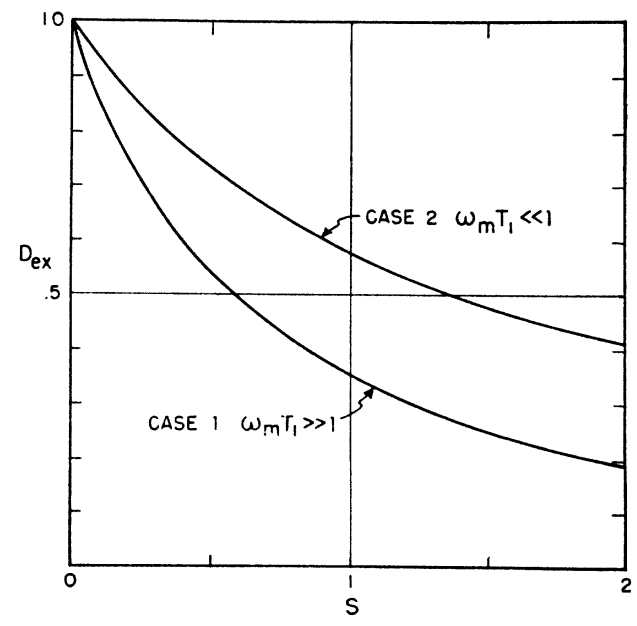

FIG. 7. Variation of the extreme output meter deflection with the saturation parameter $s$, or $\gamma^{2} H_{1}^{2} T_{1} T_{2}$, for a perfectly homogeneous field. 
paper, and is exhibited to indicate the experimental limitations. At the same time, it illustrates the enormous range which can be covered by the saturation-curve method. The horizontal shift of the curves is a measure of the relative change in $T_{1} T_{2}$. Of course, $T_{2}$ must be determined in each case by the methods already described in order to deduce the relative change in $T_{1}$. Figure 9 shows a set of saturation curves for ice at various temperatures, together with the function (18). The agreement is good enough; as a matter of fact, the difference between (17) and (18), on a logarithmic plot, is quite small, apart from a lateral shift. All saturation curves which we have observed bend downward with about the same slope.

To determine the absolute magnitude of $T_{1}$ from a saturation curve, $T_{2}$ having been measured, the relation between $H_{1}$ and the signal generator voltage must be known. The relation can be computed from the dimensions of the coil and the constants of the circuit, with which one must include the stray capacitances. We have preferred to rely on the direct measurement of $T_{1}$ for this calibration. If higher precision were required, it would be necessary to make in each case a detailed analysis like that given above for the two idealized cases 1 and 2 . The accuracy of the experimental data hardly justifies such a refinement. Fortunately, it is the relative changes in $T_{1}$, by large factors, which are most significant in the present investigation.

Discussion of the experimental results on relaxation time and line width is deferred to Sections XI to XV which follow a theoretical examination of spin-spin and spin-lattice interaction.

\section{A TRANSIENT EFFECT: THE "WIGGLES"}

Rather early in the course of this work a transient effect was noticed which merits a brief description if only because it can complicate the interpretation of nuclear absorption experiments in some cases. The effect is observed when a large amplitude of modulation is used to display the whole absorption curve on the oscilloscope, and is prominent only when $T_{1}, T_{2}$, and $1 / \gamma \delta H$ are all greater than the interval spent at resonance during the modulation cycle which is roughly $\left(H_{m} \omega_{m} \gamma T_{2}\right)^{-1}$. An example is shown in Fig. 10, a photograph of the oscilloscope trace in the case of a water sample in a very homogeneous magnetic field. The prominent "wiggles" occur always after the magnetic field has passed through resonance, and therefore appear on opposite sides of the two main peaks in Fig. 10, one of which corresponds to increasing, the other to decreasing, field.

A brief explanation of the effect is most readily given in terms of the classical model of precessing

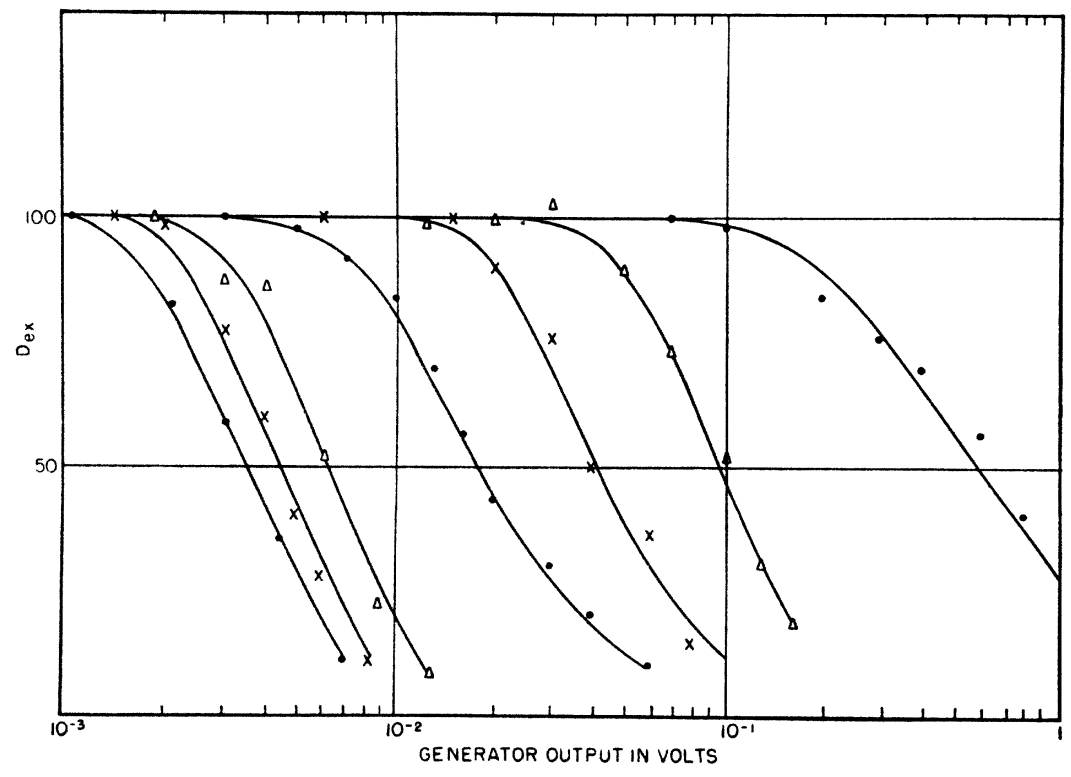

Fig. 8. Saturation curves for ferric nitrate solutions rangining in concentration from $0.0 \mathrm{~N}$ to $0.6 N$. The ordinate is the extreme output meter deflection, in arbitrary units. 
FIG. 9. Saturation curves for ice at $-35^{\circ} \mathrm{C},-17^{\circ} \mathrm{C}$, and $-5^{\circ} \mathrm{C}$. The broken curve is a plot of Eq. (18), and may be shifted horizontally to fit any of the experimental curves.

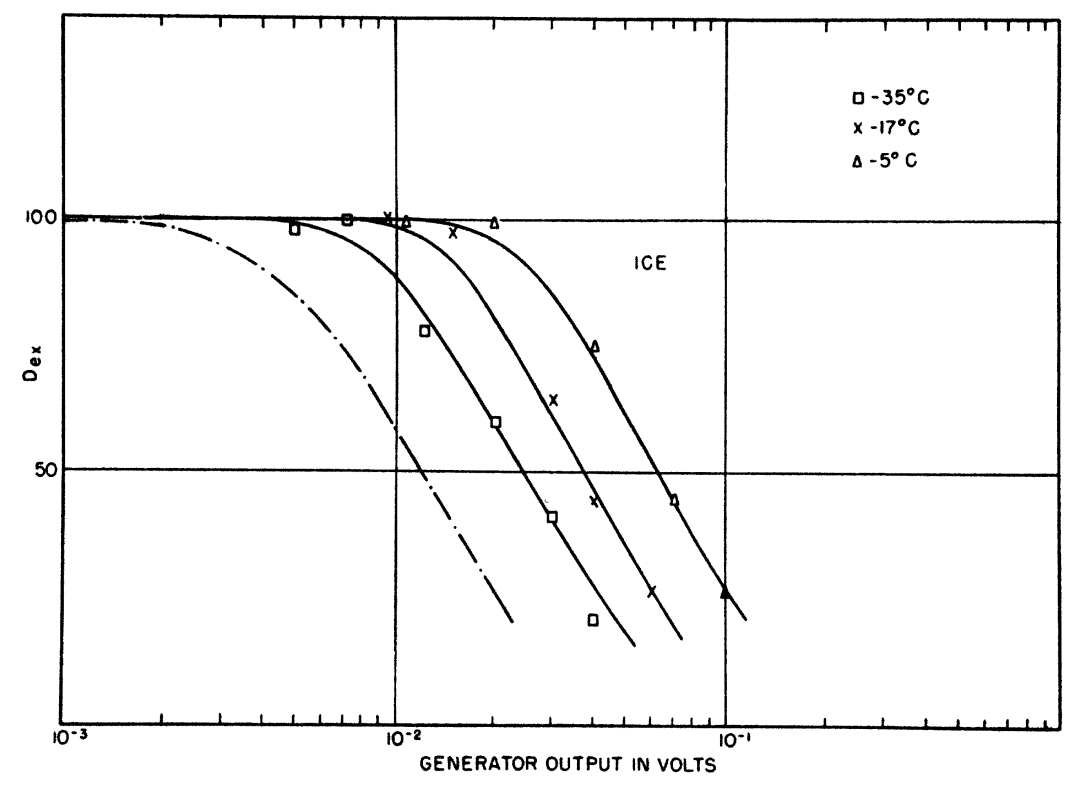

magnetic gyroscopes. Nuclear spins which have been tipped over by the application of the $r-f$ field at their precession frequency continue to precess in a coherent way after the removal of the $r-f$ field, or after the radiofrequency or magnetic field has changed to a non-resonant value. In our case the magnetic field is changing, and the nuclear precession rate, following it adiabatically, comes alternately in and out of phase with the applied signal, whose frequency has remained at what was formerly the resonance value.

The duration of the "phase memory" is limited by $T_{2}$. However, if $1 / \gamma \delta \mathrm{H}$ is smaller than $T_{2}$, interference between the effects of nuclei in different parts of the sample smears out the wiggles. By slightly detuning the receiver the wiggles on the forward trace can be enhanced and those on the back trace suppressed, or vice versa; one set of wiggles is produced by spins precessing at a rate higher than $\nu_{0}$ and the other by precession at a lower frequency. The spacing between the wiggles also varies in the manner suggested by the above explanation. A more refined treatment of the problem, which is most conveniently based on the equations of Bloch, has been made by $\mathrm{H}$. C. Torrey. ${ }^{11}$

In our measurements of $T_{1}$ by the saturation curve method, the modulation amplitude was so

\footnotetext{
${ }^{11} \mathrm{H}$. C. Torrey (private communication).
}

small that the effect was not important. The direct measurements of $T_{1}$ were made in a field sufficiently inhomogeneous to suppress the wiggles, which would not, in any case, have interfered with the interpretation of the results.

\section{SPIN-SPIN INTERACTION}

Of the processes which could conceivably bring about an exchange of energy between the spins and their surroundings, we can at once discard the interaction with the thermal radiation field. The effectiveness of this process depends upon the probability of spontaneous emission, $A$, which is of the order of $10^{-22} \mathrm{sec}^{-1}$ for a nuclear magnetic dipole transition at the frequencies with which we are concerned. The corresponding relaxation time is $T_{1}=(h \nu / k T)(1 / 2 A) \approx 10^{15}$ seconds. Although this figure must be reduced by a large factor when the nuclear system is coupled to a resonant circuit of small dimensions, ${ }^{12}$ the process is still inadequate to account for the relaxation effects observed and need not be considered further.

12 E. M. Purcell, Phys. Rev. 69, 681 (1946). In a sense, the relaxation of nuclear spins in a metal, which was considered by Heitler and Teller, reference 1, is a limiting case of the coupling of the spins to an electrical circuit and as such requires an exception to the above dismissal of radiation damping effects. Although no experimental evidence on nuclear relaxation in a metal has appeared, it is to be expected that the mechanism treated by Heitler and Teller will play an important role. 


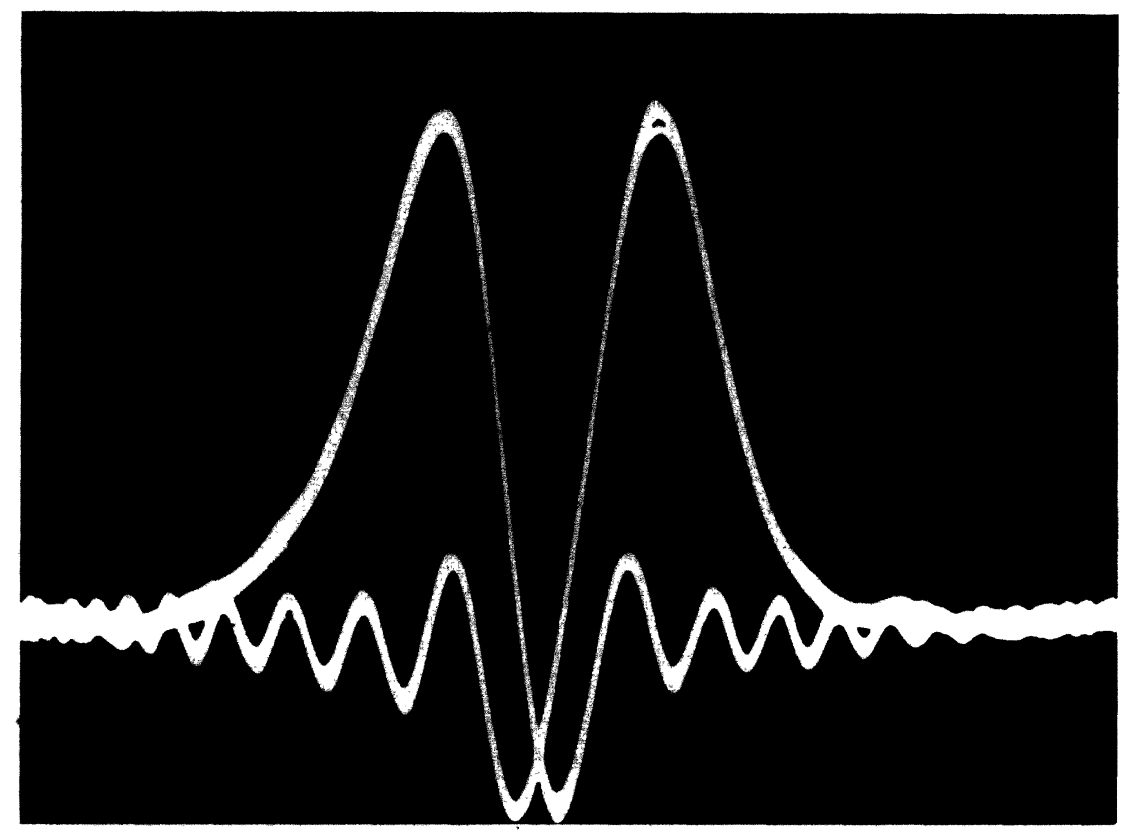

Fig. 10. An example of the transient effect described in Section IV, observed in water at $29 \mathrm{Mc} / \mathrm{sec}$. The field modulation and the oscilloscope sweep are both sinusoidal, but there is a small phase difference between them. The wiggles occur after the magnetic field has passed through resonance.

Electric forces which act during atomic collisions directly perturb the nuclear spins only by virtue of an accompanying variation in the gradient of the electric field at the nucleus and the existence of a nuclear electric quadrupole moment. Direct electrical interactions are thus reduced to a rather minor role, quite in contrast to the situation in atomic and molecular spectroscopy, and are entirely absent if the nucleus has no quadrupole moment. We shall examine one or two cases in which the nuclear quadrupoleinhomogeneous electric field interaction is the important one, but we have been concerned chiefly with nuclei of spin $\frac{1}{2}$ for which the quadrupole moment vanishes.

Magnetic interactions will occur between the nuclear magnetic moments and magnetic moments associated with electronic states, and among the nuclear magnetic moments themselves. We shall consider the nuclear dipoledipole interaction first, and in greatest detail, not only because it proves to be the dominant effect in most diamagnetic substances, so far as spin-lattice relaxation is concerned, but because it is entirely responsible for the spin-spin relaxation phenomena. In fact, we shall begin by considering a spatially rigid lattice of dipolesa model necessarily devoid of spin-lattice effects.

Before elaborating upon the description out- lined in the introduction in which the system of spins is characterized by individual spin quantum numbers, it is instructive to examine the problem from a somewhat more general point of view by regarding the entire lattice of $N$ spins as a single quantum-mechanical system. The total $z$ component of angular momentum is specified by $m \hbar$. The Hamiltonian of the system is

$\mathcal{F C}=\sum_{i} \gamma_{i} \hbar \mathbf{I}_{i} \cdot \mathbf{H}_{0}+\sum_{i} \gamma_{i} \hbar \mathbf{I}_{i} \cdot$

$$
\sum_{j}\left(\frac{\gamma_{j} \hbar \mathrm{I}_{j}}{r_{i j}{ }^{3}}-\frac{3 \gamma_{j} \hbar \mathrm{r}_{i j}\left(\mathrm{r}_{i j} \cdot \mathrm{I}_{j}\right)}{r_{i j}{ }^{5}}\right)
$$

The second term in (19) is the dipole-dipole interaction, and the sum over $j$ in this term is what we have called the local field, $H_{\text {loc }}$. In the absence of the dipole-dipole interaction the system displays $2 N I+1$ discrete levels, $m=-N I$, $-N I+1, \cdots+N I$, separated in energy by $\hbar \gamma H_{0}$. The levels are highly degenerate; for $I=\frac{1}{2}$, for example, the degeneracy is $2^{N} N ! /(N-2 m)$ ! $\times(N+2 m)$ !. The interaction term splits each of these levels into a group whose width in engery is of the order, $N^{\frac{1}{5}} \hbar \gamma H_{\text {loc }}$. Now $N^{\frac{1}{3}} \gamma H_{\text {loc }} \gg \gamma H_{0}$ in a practical case, so that the original level structure dissolves into what is essentially a continuum showing none of the previously prominent periodicity. Broer, ${ }^{13}$ who treated in

${ }^{13}$ L. J. F. Broer, Physica 10, 801 (1943). 
this way the general problem of paramagnetic absorption and dispersion, showed that the calculation of the absorption for such a system, assumed to have been brought somehow into thermal equilibrium at a temperature $T \gg h \nu / k$, could be reduced to the task of finding the density function of magnetic moment, $f(\nu)$, which he defined by:

$$
f(\nu) \Delta \nu=2 \sum_{\nu-\Delta \nu}^{\nu+\Delta \nu}\left|M_{p q}\right|^{2} \rho_{p q},
$$

where $M$ is the magnetic moment matrix, $p$ and $q$ refer to two levels separated in energy by $h \nu$, and $\rho_{p q}$ is the average occupation of these levels. The only rigorous procedure available at this point is the method of diagonal sums which Broer shows is capable of yielding a limited amount of information about the function $f(\nu)$, namely,

$$
\int_{0}^{\infty} f(\nu) d \nu, \quad \int_{0}^{\infty} \nu^{2} f(\nu) d \nu, \quad \text { etc. }
$$

the successively higher moments being increasingly difficult to calculate. An example of the application of the diagonal sum method to nuclear resonance absorption in crystals will be given in a forthcoming paper by J. H. Van Vleck. ${ }^{14}$ One of the results there obtained will be invoked to complete the following discussion in which we revert to a less rigorous perturbation method.

The unperturbed state of the system is specified by the quantum numbers $m_{I_{j}}$, or for brevity, $m_{j}$, of the individual spins, the total $z$ component of spin being given by $m=\sum m_{j}$. We assume that these magnetic quantum numbers still characterize a state after the introduction of the perturbation $V=\sum_{i} \sum_{j>i} V_{i j}$, where

$$
\begin{aligned}
V_{i j}=\gamma^{2} \hbar^{2} r_{i j}{ }^{-3}\left[I_{x_{i}} I_{x_{j}}\left(1-3 \alpha_{1}{ }^{2}\right)\right. \\
\\
+I_{y_{i}} I_{y_{j}}\left(1-3 \alpha_{2}{ }^{2}\right)+I_{z_{i}} I_{z_{j}}\left(1-3 \alpha_{3}{ }^{2}\right) \\
-3\left(I x_{i} I_{y_{j}}+I x_{j} I_{y_{i}}\right) \alpha_{1} \alpha_{2} \\
-3\left(I_{x_{i}} I_{z_{j}}+I I_{j} I_{z_{i}}\right) \alpha_{1} \alpha_{3} \\
\left.\quad-3\left(I_{y_{i}} I_{z_{j}}+I_{y_{j}} I_{z_{i}}\right) \alpha_{2} \alpha_{3}\right] .
\end{aligned}
$$

$V_{i j}$ is the magnetic interaction between the $i$ th

\footnotetext{
${ }^{14}$ J. H. Van Vleck, Phys. Rev. (in press).
}

and $j$ th spin; $\alpha_{1}, \alpha_{2}$, and $\alpha_{3}$ are the direction cosines of the radius vector $r$ in coordinates with the $z$ axis parallel to $H_{0}$. We now rearrange Eq. (20) so as to distinguish terms which leave the total $z$ component, $m$, unchanged, terms for which $\Delta m= \pm 1$, and terms for which $\Delta m=+2$ or -2 . This distinction is important because the terms involve a time factor $e^{i \Delta m \gamma H_{0} t}$. The terms with $\Delta m=0$ are time independent and leave the energy unchanged. We introduce the polar and azimuthal angles $\theta_{i j}$ and $\varphi_{i j}$ instead of the direction cosines, after which (20) can be written in the form:

$$
V_{i j}=\gamma^{2} \hbar^{2} r_{i j}^{-3}(A+B+C+D+E+F),
$$

where

$$
\begin{aligned}
& A=I_{z_{i}} I_{z_{j}}\left(1-3 \cos ^{2} \theta_{i j}\right), \quad(\Delta m=0), \\
& B=-\frac{1}{4}\left[\left(I_{x_{\imath}}-i I_{y_{i}}\right)\left(I_{x_{j}}+i I_{y_{j}}\right)\right. \\
& \left.+\left(I_{x_{i}}+i I_{y_{i}}\right)\left(I_{x_{j}}-i I_{y_{j}}\right)\right] \\
& \times\left(1-3 \cos ^{2} \theta_{i j}\right), \quad(\Delta m=0), \\
& C=-\frac{3}{2}\left[\left(I_{x_{i}}+i I_{y_{i}}\right) I_{z_{j}}+\left(I_{x_{j}}+i I_{y_{j}}\right) I_{z_{i}}\right] \\
& \times \sin \theta_{i j} \cos \theta_{i j} e^{-i \varphi_{i j}} e^{i \gamma H_{0} t}, \quad(\Delta m=1), \\
& D=-\frac{3}{2}\left[\left(I_{x_{i}}-i I_{y_{i}}\right) I_{z_{j}}+\left(I_{x_{j}}-i I_{y_{j}}\right) I_{z_{i}}\right] \sin \theta_{i j} \\
& \times \cos \theta_{i j} e^{+i \varphi_{i j}} e^{-i \gamma H_{0} t}, \quad(\Delta m=-1), \\
& E=-\frac{3}{4}\left(I_{x_{i}}+i I_{y_{i}}\right)\left(I_{x_{j}}+i I_{y_{j}}\right) \sin ^{2} \theta_{i j} \\
& \times e^{-2 i \varphi_{i j} e^{+2 i \gamma H_{0} t}, \quad(\Delta m=2),} \\
& F=-\frac{3}{4}\left(I_{x_{i}}-i I_{y_{i}}\right)\left(I_{x_{j}}-i I_{y_{j}}\right) \sin ^{2} \theta_{i j} \\
& \times e^{+2 i \varphi_{i j} e^{-2 i \gamma H_{0} t} .}(\Delta m=-2) .
\end{aligned}
$$

Terms $A$ and $B$ give rise to secular perturbations; terms $C$ to $F$ represent periodic perturbations of small amplitude. That these last four terms cannot cause appreciable changes is a consequence of the conservation of energy. For our rigid-lattice model, then, only terms $A$ and $B$ need be considered. Classically $A$ corresponds to the change in the $z$ component of the magnetic field by the $z$ components of the neighboring magnetic moments. This local field will vary from nucleus to nucleus, slightly changing the Larmor frequency of each. Suppose that the $i$ th nucleus is surrounded by a number of neighbors, which for simplicity we shall assume to have spin $I=\frac{1}{2}$. Each of these can be parallel or 
antiparallel to $H_{0}$, and every arrangement leads to some value of the static part of the local field. From the nearest neighbors alone, we will usually obtain several possible values; each of these will be further split when the next nearest neighbors are taken into account, and so on. The result will be a continuum of possible values of the local static field, centered on $H_{0}$, whose shape would have to be calculated for the particular spatial configuration of the lattice involved, and the given direction of $H_{0}$. The calculation is simplified, in all cases of interest, by the near equality of probability for either spin orientation, which also insures the symmetry of the distribution about $H_{0}$. We have carried out the calculation for a special case of a cubic lattice of spins, obtaining a roughly Gaussian shape for the distribution. In many cases an approximately Gaussian shape is to be expected. An important exception is found, however, when the nuclei lie near together in pairs; several such cases have been studied experimentally by G. E. Pake and are treated in a forthcoming paper. ${ }^{15}$

We can obtain an estimate of the line width by calculating the mean square contribution of each neighbor separately and taking the square root of the sum of these contributions, thereby assuming that the orientation of each spin is independent of that of another. The contribution of term $A$ to the mean square local field is given by

$$
\begin{aligned}
\left\langle H_{\mathrm{loc}}{ }^{2}\right\rangle_{\mathrm{Av}}=\frac{1}{3} \gamma^{2} \hbar^{2} I(I+1) & \\
& \times \sum_{j}\left(1-3 \cos ^{2} \theta_{\imath j}\right)^{2} \gamma_{i j}{ }^{-6},
\end{aligned}
$$

and the mean square deviation in frequency by

$$
\left\langle\Delta \omega^{2}\right\rangle_{\mathrm{Av}}=\gamma^{2}\left\langle H_{\mathrm{loc}}{ }^{2}\right\rangle_{\mathrm{Av}}
$$

To this we must add the contribution of $\operatorname{term} B$, which corresponds to the simultaneous flopping of two antiparallel spins $\left(\Delta m_{i}=+1\right.$ and $\Delta m_{j}$ $=-1$, or vice versa). Such a process is energetically possible and is caused by the precession of the spins around $H_{0}$ with the Larmor frequency; the precession of one spin produces a rotating field at the resonance frequency at the location of another, resulting in reciprocal transitions. We may say that this process limits the lifetime

\footnotetext{
${ }^{15}$ G. E. Pake, J. Chem. Phys. (in press).
}

of a spin in a given state and therefore broadens the spectral line. The effect is taken into account by applying the proper numerical factor to (24). The factor has been calculated by Van Vleck by the diagonal sum method.14 In the case of a lattice of identical spins it is $9 / 4$, the r.m.s. (angular) frequency deviation including both effects then being,

$$
\begin{aligned}
{\left[\left\langle\Delta \omega^{2}\right\rangle_{\mathrm{Av}}\right]^{\frac{1}{2}} \equiv\left(1 / T_{2}{ }^{\prime \prime}\right)=\frac{3}{2} \gamma^{2} \hbar[I(I+1) / 3]^{\frac{1}{2}} } \\
\\
\times\left[\sum_{j \neq i}\left(1-3 \cos ^{2} \theta_{i j}\right)^{2} r_{i j}{ }^{-6}\right]^{\frac{1}{2}} .
\end{aligned}
$$

If the nuclei are not identical in gyromagnetic ratio, neighbors which are dissimilar to the nucleus $i$ contribute only through the term $A$, and the sum over $j$ in (25) has to be modified in an obvious way.

We have here introduced the quantity $T_{2}{ }^{\prime \prime}$, defined above, as a specification of the line width in this limiting case of a rigid lattice. We shall later use $T_{2}{ }^{\prime}$ to describe the effect upon the line width of spin-spin interactions as modified by lattice motion, reserving the unprimed $T_{2}$ for the specification of the line width in the general case, to which the spin-lattice interaction also contributes. If the line shape is Gaussian, the quantity $2 / T_{2}{ }^{\prime \prime}$ is the width between points of maximum slope, on an $\omega$ scale. In calculating the maximum absorption for such a line shape, in the rigid lattice, the $T_{2}$ defined by Eq. (14) is to be identified with $(\pi / 2)^{\frac{1}{2}} T_{2}^{\prime \prime}$.

An accurate experimental test of (25) requires, of course, a determination of the line shape. A substance which the rigid lattice model might be expected to represent fairly well is fluorite, $\mathrm{CaF}_{2}$. The measurements of the $\mathrm{F}^{19}$ resonance line in $\mathrm{CaF}_{2}$, previously reported, ${ }^{16}$ and subsequent unpublished work on the same substance by G. E. Pake, are in good agreement with Eq. (25) (see reference 14).

\section{SPIN-LATTICE INTERACTION}

The rigid lattice model does not exhibit thermal relaxation of the spin system as a whole. We shall now show that if the position coordinates, $r_{i j}, \theta_{i j}$, and $\varphi_{i j}$ are made to vary with time, transitions are induced in which $m$ changes, the spin system and the "lattice" thereby ex-

${ }^{16}$ E. M. Purcell, N. Bloembergen, and R. V. Pound, Phys. Rev. 70, 988 (1946). 
changing energy. Not only does this provide thermal contact between the spins and an adequate heat reservoir, it also modifies, in some cases drastically, the spin-spin interaction itself.

Waller ${ }^{17}$ in 1932 treated the interaction of spins with lattice vibrations; his theory was applied by Heitler and Teller ${ }^{1}$ to estimate the effectiveness of such an interaction in bringing about thermal equilibrium of a nuclear spin system. We want to include more general types of motion, in particular, what we may loosely call Brownian motion in a liquid or non-crystalline solid. It will turn out that such internal degrees of freedom, when active, are far more effective as a source of spin-lattice interaction than are lattice vibrations, and play an important role in substances which by many standards would be regarded as crystalline.

As far as the Hamiltonian for the nuclear spin system is concerned, we regard the motion of the molecules as produced by external forces, the atomic interactions being mainly of an electrical nature. The atom or molecule is simply a vehicle by which the nucleus is conveyed from point to point. We thus neglect the reaction of the magnetic moments of the nuclei upon the motion. The factors in (22) which contain the position coordinates are now to be expanded in Fourier integrals. We want to distinguish in our complex notation between positive and negative frequencies, and we therefore define the intensities $J(\nu)$ of the Fourier spectra of the three position functions involved by

$$
\begin{aligned}
\left\langle\sum_{j}\left|\left(1-3 \cos ^{2} \theta_{i j}(t)\right) r_{i j}{ }^{-3}(t)\right|^{2}\right\rangle_{\mathrm{Av}} & \\
& =\int_{-\infty}^{\infty} J_{0}(\nu) d \nu, \\
\left\langle\sum_{j}\left|\sin \theta_{i j}(t) \cos \theta_{i j}(t) e^{i \varphi i j(t)} r_{i j}{ }^{-3}(t)\right|{ }^{2}\right\rangle_{\mathrm{AV}} & \\
& =\int_{-\infty}^{\infty} J_{1}(\nu) d \nu, \\
\left\langle\sum_{j}\left|\sin ^{2} \theta_{i j} e^{2 i \varphi i j(t)} r_{i j}{ }^{-3}(t)\right|^{2}\right\rangle_{\mathrm{Av}} & =\int_{-\infty}^{\infty} J_{2}(\nu) d \nu .
\end{aligned}
$$

Reconsidering now the terms $C, D, E$, and $F$ in the expression for the perturbation (22), we observe that if $J_{1}\left(-\nu_{0}\right)$ and $J_{1}\left(+\nu_{0}\right)$, where

${ }^{17}$ I. Waller, Zeits. f. Physik 79, 370 (1932).
$2 \pi \nu_{0}=\gamma H_{0}$, are different from zero, $C$ and $D$ become secular perturbations because the time factor cancels out. Similarly, $E$ and $F$ become secular perturbations if $J_{2}\left(-2 \nu_{0}\right)$ and $J_{2}\left(+2 \nu_{0}\right)$ do not vanish. Transitions are thus made possible in which $m$ changes by \pm 1 or \pm 2 , the thermal motion providing, or absorbing, the requisite energy.

We can form a simple picture of the processes involved by recalling that the local field at one nucleus $i$ due to a neighbor $j$, in the rigid-lattice model, consists of two parts, a static part depending on $I_{z_{j}}$ and a rotating part depending on $\left(I_{x_{j}}-i I_{y_{j}}\right)$. The motion of the neighbor $j$ now causes the field arising from its $z$ component of magnetic moment to fluctuate; if the motion contains frequencies synchronous with the precession of the neighbor $i$, the nucleus $i$ will find itself exposed to a radiofrequency field capable of inducing a transition. This is the effect described by terms $C$ and $D$. The doublefrequency effect comes about as follows: the field at $i$ which arises from the precessing components of magnetic moment of $j$ consists in general of both right and left circularly polarized components. In the rigid lattice one of these has a negligible effect while the one which rotates in the same sense as the precession of $i$ causes the perturbation expressed by term $B$. If, however, we impart to the nucleus $j$ a suitable motion, at the frequency $2 \nu_{0}$ we can reverse the sense, at the nucleus $i$, of the originally ineffective circular component, thus permitting it to interact with $i$. An exactly similar situation will necessarily be created at the nucleus $j$, with regard to the field arising from the precessing components of moment of $i$, hence a double transition occurs. This also shows why still higher multiples of $\nu_{0}$ do not enter the problem.

We can now compute the probability for a change $\Delta m=+1$ in the magnetic quantum number of the $i$ th spin brought about by the fields associated with the "local field spectrum." By a perturbation calculation, using terms $C$ and $E$, and taking an average over all values $m_{j}$ of the neighbors, we find for the transition probability,

$W_{m_{i} \rightarrow m_{i}+1}=\frac{3}{4} \gamma^{4} \hbar^{2}\left(I-m_{i}\right)\left(I+m_{i}+1\right) I(I+1)$

$$
\times\left[J_{1}\left(-\nu_{0}\right)+\frac{1}{2} J_{2}\left(-2 \nu_{0}\right)\right] .
$$


If the values of $I$ and $\gamma$ are not the same for all nuclei, Eq. (27) can,be generalized as follows:

$$
\begin{aligned}
& W_{m_{i} \rightarrow m_{i}+1}=\frac{3}{4} \gamma_{i}{ }^{2} \hbar^{2}\left(I_{i}-m_{i}\right)\left(I_{i}+m_{i}+1\right) \\
& \times \sum_{j} \gamma_{j}{ }^{2} I_{j}\left(I_{j}+1\right) \\
& \quad \times\left[J_{1 j}\left(-\nu_{0_{i}}\right)+\frac{1}{2} J_{2 j}\left(-\nu_{0 j}-\nu_{0_{i}}\right)\right],
\end{aligned}
$$

where

$$
\nu 0_{j}=\gamma_{j} H_{0} / 2 \pi .
$$

The result can be extended to include the effect of perturbing fields arising from other dipole sources such as electronic spins. In such cases the quantization of these spins may be time dependent under the influence of external forces (e.g., paramagnetic relaxation phenomena involving electronic spins) and the function $\left[\left(I_{j}-m_{j}(t)\right)\left(I_{j}+m_{j}(t)+1\right)\right]^{\frac{1}{2}}$ must be included with the position functions when the Fourier expansion is performed, the factor $I_{j}\left(I_{j}+1\right)$ in (28) then being dropped.

The expressions for transitions with $\Delta m_{i}=-1$ are, of course, the same except that the intensities have to be taken at positive frequencies in the Fourier spectrum. Since $J(\nu)$ will in all cases be an even function of $\nu$, it would appear that the motion should produce as many transitions up as down. However, the transition probabilities must be weighted by the Boltzmann factors of the final states, as required by the fact that the system left to itself will come to thermal equilibrium and the principle of detailed balancing. That is, if $N_{p}$ and $N_{q}$ are the equilibrium populations of levels $p$ and $q$ which differ in energy by $E_{p}-E_{q}$, we must have, in equilibrium,

or

$$
\begin{gathered}
N_{p} W_{p \rightarrow q}=N_{q} W_{q \rightarrow p}, \\
W_{p \rightarrow q} / W_{q \rightarrow p}=N_{q} / N_{p}=e^{\left(E_{p}-E_{q}\right) / k T} .
\end{gathered}
$$

But the probability of a single transition from $p$ to $q$, in a system such as we are considering, cannot depend on the population of $q$; it is therefore necessary to suppose that the $W$ 's are related by the Boltzmann factor of Eq. (29) even when the spins are not in equilibrium with the lattice, $T$ being the lattice temperature. We are thus enabled to trace the approach to equilibrium of the spin system coupled to a reservoir which remains throughout the process at the temperature $T$. This last assumption is justified if $k T \gg \gamma H_{0} \hbar$ for the specific heat of the lattice is then very much greater than that of the spins.

Consider first the case $I=\frac{1}{2}$. Let the total number of spins be $N$, with $N_{+}$and $N_{-}$denoting the population of the lower and upper state, respectively. For $W_{+\rightarrow-}$ and $W_{-\rightarrow+}$ we take $W$ as given by Eq. (27) modified by the appropriate Holtzmann factors :

$$
\begin{aligned}
& W_{+\rightarrow-}=W e^{-\gamma H_{0} \hbar / 2 k T} \approx W\left[1-\left(\gamma \hbar H_{0} / 2 k T\right)\right] ; \\
& W_{-\rightarrow+} \approx W\left[1+\left(\gamma \hbar H_{0} / 2 k T\right)\right] \\
& \text { with } \\
& W=(9 / 16) \gamma^{4} \hbar^{2}\left[J_{1}\left(\nu_{0}\right)+\frac{1}{2} J_{2}\left(2 \nu_{0}\right)\right] .
\end{aligned}
$$

Writing $n$ for $N_{+}-N_{-}$, the surplus in the lower state, we have

$$
\begin{aligned}
d n / d t=2 N_{-} W_{-\rightarrow+}-2 N_{+} W_{+\rightarrow-} \\
=(N+n) W_{-\rightarrow+}-(N-n) W_{+\rightarrow} .
\end{aligned}
$$

If we assume that $|n| \ll N$, as it will be in all cases of present interest, and denote by $n_{0}$ $=N \gamma \hbar H_{0} / 2 k T$ the equilibrium value of $n$, we have

$$
d n / d t=2 W\left(n_{0}-n\right),
$$

showing that equilibrium is approached according to a simple exponential law with a characteristic time

$$
T_{1}=1 / 2 W .
$$

We thus make connection with, and justify, the manner in which the spin-lattice relaxation time $T_{1}$ was introduced in Eq. (11).

For $I=\frac{1}{2}$ it is always possible to define a spin temperature $T_{s}$ through $e^{\gamma \hbar H_{0} / k T_{s}}=N_{-} / N_{+}$, and the approach to equilibrium can be described as a cooling, or warming, of the spin system as a whole. The corresponding differential equation, in which $T_{L}$ is the lattice temperature, is

$$
d T_{s} / d t=\left(1 / T_{1}\right)\left(T_{s} / T_{L}\right)\left(T_{L}-T_{s}\right) .
$$

Clearly the transient behavior of the spin system is more simply described by the reciprocal temperature, or by $n$, than by the temperature directly.

The situation is more complicated when $I>\frac{1}{2}$. A spin temperature cannot be defined unless the populations of successive levels are related by the same factor. Corresponding to this additional arbitrariness in the initial state of the system, 
Eq. (32) has to be replaced by a number of simultaneous differential equations. However, a detailed examination of this question which can be found elsewhere, ${ }^{18}$ leads to a simple result when the initial state of the spin system happens to be describable by a temperature $T_{s}$, and when $k T_{s} \gg \gamma \hbar H_{0} \ll k T_{L}$. The approach to equilibrium then resembles that of the $I=\frac{1}{2}$ system, the surplus number in each level with respect to the next above increasing or diminishing with the same characteristic time. Any intermediate state of the system can then be assigned a temperature, and (32) applies. It is found, moreover, that value of $W$ which must then be used in (33) is

$$
\frac{3}{4} \gamma^{4} h^{2} I(I+1)\left[J_{1}\left(\nu_{0}\right)+\frac{1}{2} J_{2}\left(2 \nu_{0}\right)\right] .
$$

That is to say, the spin-lattice relaxation time for nuclei of spin $I$ exposed to a given local field is the same as the relaxation time for nuclei of the same gyromagnetic ratio $\gamma$, but with $I=\frac{1}{2}$, exposed to the same field.

The above argument would apply, for example, to a spin system with $I>\frac{1}{2}$ which is allowed to come to equilibrium in a field $H_{0}$, after which $H_{0}$ is changed adiabatically to a new value. A unique $T_{1}$ can be defined which measures the rate of approach of the populations, $N_{-I}$, $\cdots N_{I}$, to their new equilibrium distribution. Similarly, if the application of an intense radiofrequency field of frequency $\nu_{0}$ has completely equalized the level populations, the recovery from such saturation (infinite spin temperature) follows the same law.

In general, however, the application of a radiofrequency field alters the distribution to one which is not precisely a Boltzmann distribution-the spin system is itself no longer in equilibrium. The most powerful agency tending to restore internal equilibrium among the spins is in many cases the spin-spin interaction. By means of the process associated with the term $B$ in Eq. (22), in which $\Delta m_{i}=+1, \Delta m_{j}=-1$, or vice versa, the level populations can be readjusted. If $I=1$, for example, transitions of the type $-1 \rightarrow 0$ (for $m_{i}$ ) accompanied by $+1 \rightarrow 0$ (for $m_{j}$ ) augment the population of the central level, while the reverse double transition depletes it. If $N_{-1}, N_{0}, N_{+1}$ are the populations of the levels, the rate at which transitions of the first type occur is proportional to $N_{-1} \cdot N_{\rightarrow 1}$, while the rate at which the second type occur is proportional to $N_{0}^{2}$, but otherwise identical. A stationary state is therefore reached only when $N_{0}{ }^{2}=N_{-1} \cdot N_{+1}$, which is precisely the description of a Boltzmann distribution over three equidistant levels.

With the above reservations concerning the case $I>\frac{1}{2}$, we now write the general formula for the spin-lattice relaxation due to dipole-dipole interaction in the case of identical nuclei. $\$ \ddagger$

$$
\begin{aligned}
& \left(1 / T_{1}\right)=\frac{3}{2} \gamma^{4} \hbar^{2} I(I+1)\left[J_{1}\left(\nu_{0}\right)+\frac{1}{2} J_{2}\left(2 \nu_{0}\right)\right] . \\
& \text { vIII. EFFECT OF THE MOTION UPON THE } \\
& \text { LINE WIDTH }
\end{aligned}
$$

\section{EFFECT OF THE MOTION UPON THE}

The terms $A$ and $B$ of Eq. (22) must now be reconsidered to see what effect the motion of the nuclei has upon the line width. These terms will still represent secular perturbations if we take the components near zero frequency in $J_{0}(\nu)$, the intensity of the Fourier spectrum of $\sum_{j}\left(1-3 \cos ^{2} \theta_{i j}(t)\right) r_{i j}{ }^{-3}(t)$. The question is, which frequencies are to be considered as "near zero." We may say that the perturbation is secular up to the frequency $\nu$ for which $h \nu$ is of the same order as the actual splitting of the energy levels by the perturbation. The resulting line width will be specified by $\Delta \omega=2 \pi \Delta \nu=1 / T_{2}{ }^{\prime}$, and with the application of the above criterion, $T_{2}{ }^{\prime}$ is to be found from the following modification of Eq. (34) :

$$
\begin{aligned}
& 1 / T_{2}^{\prime}=\frac{3}{2} \gamma^{2} \hbar[I(I+1) / 3]^{\frac{1}{3}} \\
& \qquad \times\left[\int_{-1 / \pi T_{2}^{\prime}}^{+1 / \pi T_{2}^{\prime}} J_{0}(\nu) d \nu\right]^{\frac{1}{2}} .
\end{aligned}
$$

Again an exception must be noted in the case of non-identical nuclei. If $\gamma_{i} \neq \gamma_{j}$, the secular perturbation from term $B$ depends on the intensity of the $J_{0}$ spectrum in the neighborhood of $\nu=\left(\gamma_{i}-\gamma_{j}\right) H_{0} / 2 \pi$ rather than $\nu=0$.

The observed line width is not determined by $T_{2}^{\prime}$ alone because the spin-lattice relaxation effected by the terms $C$ to $F$ limits the lifetime of the nucleus in a given state. For $I=\frac{1}{2}$, the mean lifetime in each of the two levels is $1 / W$, or $2 T_{1}$. The resulting line width, for two levels

$\ddagger$ The case of non-identical nuclei calls for a rather obvious generalization which we omit. 
broadened in this way, is, by a well-known argument $^{19}$ twice the width of one level, the line shape being given to a high degree of approximation, $\left(T_{1} \gg 1 / \nu_{0}\right)$ by

$$
g_{1}(\nu)=\frac{4 T_{1}}{1+16 \pi^{2} T_{1}^{2}\left(\nu-\nu_{0}\right)^{2}}
$$

a curve whose width between half-value points is $1 / 2 \pi T_{1}$ on a frequency scale. With this must be combined the distribution resulting from the perturbations $A$ and $B$, which, if it had a Gaussian shape, would be

$$
g_{2}(\nu)=T_{2}{ }^{\prime}(2 \pi)^{-\frac{1}{2}} \exp \left[-2 \pi^{2}\left(\nu-\nu_{0}\right)^{2} T_{2}{ }^{2}\right] \text {. }
$$

Roughly speaking, we can say that the combination of two bell-shaped curves will yield another bell-shaped curve the width of which is about the sum of the widths of the composing curves. Thus,

$$
\left(1 / T_{2}\right)=(2 / \pi)^{\frac{1}{2}}\left(1 / T_{2}{ }^{\prime}\right)+\left(1 / 2 T_{1}\right) .
$$

We cannot attach a precise meaning to $T_{2}$ in general, but the numerical factors in (38) have been chosen $\ddagger \ddagger \ddagger$ with two limiting cases in mind: (a) $T_{2}{ }^{\prime} \ll T_{1}$, and (b) $T_{2}{ }^{\prime} \approx T_{1}$. In the former case the factor $(2 / \pi)^{\frac{1}{3}}$ is consistent with Eq. (14) for the Gaussian curve to which, in this case, we may expect the line to bear some resemblance. The last term in (38), which is important in case (b), is correctly written for the damped oscillator line shape. However, in this case, an additional uncertainty arises from the arbitrariness of the choice of limits in Eq. (35).

\section{THE SPECTRUM OF THE SPACE COORDINATES}

In order to apply this general theory of relaxation time and line width to a particular substance, it is necessary to develop the Fourier spectra of the functions of position coordinates,

$$
\begin{aligned}
& F_{0 j}=\left(1-3 \cos ^{2} \theta_{i j}\right) r_{i j}{ }^{-3}, \\
& F_{1 j}=\sin \theta_{i j} \cos \theta_{i j} e^{i \varphi i r_{i j}{ }^{-3},} \\
& F_{2 j}=\sin ^{2} \theta_{i j} e^{2 i \varphi i j \gamma_{i j}}{ }^{-3},
\end{aligned}
$$

${ }^{19} \mathrm{~V}$. Weisskopf and E. Wigner, Zeits. f. Physik 63, 54 (1930); 65, 18 (1930).

$\ddagger \ddagger$ It seems worth while to preserve internal consistency among the definitions and theoretical formulas even at the risk of making the formulas appear less approximate than they really are. from which to obtain the functions $J_{0 j}(\nu), J_{1 j}(\nu)$, $J_{2 j}(\nu)$ governing the intensity of the local field spectrum. By postulating thermal vibrations in an otherwise rigid lattice, for example, one is led directly to Waller's result for spin-lattice relaxation in a crystal. Our interest here is in liquids, for which the functions $F$ will vary in a random fashion with time, as the particles containing the magnetic nuclei take part in the Brownian motion. The motion will be isotropic on the average; hence, as is at once evident from (39), the time average of each of the functions $F$ is zero.

The statistical character of the motion justifies an assumption customary in the theory of fluctuation phenomena, that

$$
\left\langle F(t) F^{*}(t+\tau)\right\rangle_{\mathrm{Av}}=K(\tau) .
$$

The right side of $(40)$ is called the correlation function of $F(t)$. It is assumed to be an even function of $\tau$ and independent of $t$. It follows immediately that $K(\tau)$ is real. We may assume also that $K(\tau)$ approaches zero for sufficiently large values of $\tau$. The relation ${ }^{20}$ between the Fourier spectrum of a function $F$ and the correlation function of $F$ enables us to write for the spectral intensity,

$$
J(\nu)=\int_{-\infty}^{\infty} K(\tau) e^{2 \pi i \nu \tau} d \tau .
$$

Since $K(\tau)$ is real and even, $J(\nu)$ is real and even.

In order to simplify the following discussion we make an assumption about the form of $K(\tau)$, namely, that

$$
K(\tau)=\left\langle F(t) F^{*}(t)\right\rangle_{\mathrm{Av}} e^{-|\tau| / \tau_{c}} .
$$

The time $\tau_{c}$ is a characteristic of the random motion and the function whose correlation $K$ measures, and is called the correlation time. Temporarily we shall assume that $\tau_{c}$ is the same for each of the functions $F_{0}, F_{1}$, and $F_{2}$, returning to this question later. From (41) and (42) we find, for each of the three $J$ 's,

$$
J(\nu)=\left\langle F(t) F^{*}(t)\right\rangle_{\mathrm{Av}} 2 \tau_{c}\left(1+4 \pi^{2} \nu^{2} \tau_{c}^{2}\right)^{-1} .
$$

The intensity in the spectrum is nearly constant

${ }^{20}$ See, for example, Ming Chen Wang and G. E. Uhlenbeck, Rev. Mod. Phys. 17, 323 (1945). 
at very low frequencies but falls off rapidly when $2 \pi \nu \tau_{c} \gg 1$. The subscript $j$ has been omitted above, but it will be understood that, in general, not all neighbors will have the same correlation time.

\section{THE THEORY APPLIED TO WATER}

The application of the preceding theory will now be illustrated by a calculation of the relaxation time for protons in water. A given proton has as its nearest neighbor the other proton in the $\mathrm{H}_{2} \mathrm{O}$ molecule, and we consider first the effect of this exceptional neighbor. Vibration of the molecule can be neglected, because of its high frequency and small amplitude. We regard the molecule as rigid, and assume that the orientation of the vector connecting the two protons varies randomly, no direction being preferred. Only the angle coordinates are now variable in time, the functions $F$ being

$$
\begin{aligned}
& F_{0}=\left(1-3 \cos ^{2} \theta\right) / b^{3}, \\
& F_{1}=\cos \theta \sin \theta e^{i \varphi} / b^{3}, \\
& F_{2}=\sin ^{2} \theta e^{2 i \varphi} / b^{3},
\end{aligned}
$$

where $b$ is the interproton distance. We can substitute a statistical average over the spatial coordinates for the time average specified in (43). The averages are readily found:

$$
\begin{aligned}
& \left\langle F_{0}(t) F_{0}^{*}(t)\right\rangle_{\mathrm{AV}}=4 / 5 b^{6} ; \\
& \left\langle F_{1}(t) F_{1}{ }^{*}(t)\right\rangle_{\mathrm{AV}}=2 / 15 b^{6} ; \\
& \left\langle F_{2}(t) F_{2}{ }^{*}(t)\right\rangle_{\mathrm{AV}}=8 / 15 b^{6} .
\end{aligned}
$$

Returning to the general formula (34) with (43) and (45) we obtain for the relaxation time (involving the nearest neighbor only)

$$
\begin{array}{r}
\left(1 / T_{1}\right)=(3 / 10)\left(\gamma^{4} h^{2} / b^{6}\right)\left[\tau_{c} /\left(1+4 \pi^{2} \nu_{0}^{2} \tau_{c}^{2}\right)\right. \\
\left.+2 \tau_{c} /\left(1+16 \pi^{2} \nu_{0}^{2} \tau_{c}^{2}\right)\right] .
\end{array}
$$

The determination of the correlation time $\tau_{c}$ is closely related to the problem encountered in the Debye theory of dielectric dispersion in polar liquids; there also it is necessary to estimate the time, $\tau$, during which molecular orientation persists. ${ }^{21}$ Debye assumed that in the first approximation the molecule could be treated as

${ }^{21}$ P. Debye, Polar Molecules (Dover Publications, New York, 1945), Chapter V. a sphere of radius $a$ imbedded in a viscous liquid, and thereby obtained the relation $\tau=4 \pi \eta a^{3} / k T$, in which $\eta$ is the viscosity of the liquid. We adopt the same procedure here, noting one difference between the two problems-a rather unimportant difference, to be sure, in view of the much over-simplified model, but a characteristic one. The function whose correlation time is required in the Debye theory is $\cos \theta$, the symmetry character of which is essentially different from that of the functions $F_{0}, F_{1}$, and $F_{2}$ with which we are concerned. Because of this the correlation time for a given model 9 will not be the same in our case. In fact, for the sphere in a viscous liquid it is, for each $\uparrow$ T of the functions $F$,

$$
\tau=4 \pi \eta a^{3} / 3 k T \text {. }
$$

Adopting Eq. (47) and assuming that $a=1.5$ $\times 10^{-8} \mathrm{~cm}$, we find for water at $20^{\circ} \mathrm{C}(\eta=0.01$ poise) : $\tau_{c}=0.35 \times 10^{-11}$ second. For comparison we note that the measurements by Saxton ${ }^{22}$ of the dielectric constant of water in the microwave region lead to a value $0.81 \times 10^{-11}$ seconds for the Debye characteristic time, at $20^{\circ} \mathrm{C}$. Although this is somewhat less than 3 times our estimated $\tau_{c}$, we must remember that with further refinement of the molecular model a difference between $\tau_{c}$ and $1 / 3 \tau_{\text {Debye }}$ would arise from the fact that the $H-H$ line and the polar axis of the molecule lie in different directions.

We note at once that $2 \pi \nu \tau_{c} \ll 1$, so that the term in brackets in Eq. (46) becomes simply $3 \tau_{c}$. In other words, we find ourselves at the low frequency end of the local field spectrum, where the spectral intensity is independent of $\nu$ and directly proportional to $\tau_{c}$. For the interproton

T Another model, in some ways a better representation of the molecule and its environment, is one in which only a limited number of orientations are permitted, the transitions from one to another taking place in a "jump." Imagine, for example, that the molecular axis-in our case the $H-H$ line-can point toward any of the eight corners of a cube centered at the molecule, transitions taking place with the probability $w$ between any such direction and one of the three adjacent directions only. For this model the correlation functions of $F_{0}, F_{1}$, and $F_{2}$ are all of the form (42) with the correlation time $3 / 4 w$, whereas the correlation time of $\cos \theta$ is $3 / 2 w$.

IT Note that $F_{0}, F_{1}$, and $F_{2}$ belong to the same spherical harmonic, $Y_{2}(\theta, \varphi)$. It can be shown that the correlation function of $Y_{l}(\theta, \varphi)$ for the sphere in a viscous liquid, is $\exp \left[-l(l+1) k T \tau / 8 \pi \eta a^{3}\right]$.

${ }_{22}^{2}$ J. A. Saxton, Radio Research Board Report C115 (declassified), D.S.I.R., March 20, 1945. (This is a British report.) 
distance we take $1.5 \times 10^{-8} \mathrm{~cm}$. Upon substituting numbers into (46), we obtain for $\left(1 / T_{1}\right)_{p}$ the relaxation rate due to the proton partner only, 0.19 sec. $^{-1}$.

The effect of the neighboring molecules must now be estimated. The fluctuation in the local field arising from the neighbors is mainly caused by their translational motion, and only to a minor extent by their rotation. We regard the molecules as independent, attributing to each either a magnetic moment of $2 \mu_{p}$ and spin $I=1$, of else zero spin and moment. Three-fourths of the neighboring molecules are of the former (ortho) type. We ask for $\left\langle F(t) F^{*}(t)\right\rangle_{A v}$ and $\tau_{c}$ for the molecules in a spherical shell between $r$ and $r+d r$. The center of the shell is located at, and moves with, the molecule containing the proton $i$. A reasonable choice for $\tau_{c}$ is the time it takes the molecule $j$ to move a distance $r$ in any direction from its original position on the shell, for in that time the field at the proton $i$, due to $j$, will have changed considerably. The relative motion of $i$ and $j$ is simply diffusion, and can be described most directly by means of the diffusion coefficient $D$ of the liquid. Applying the above criterion for $\tau_{c}$, and remembering that both molecules diffuse, we find,

$$
\tau_{0}=r^{2} / 12 D .
$$

To find $\left\langle F(t) F^{*}(t)\right\rangle_{\mathrm{Av}}$ the angular functions are averaged as before and then, treating the molecules as independent, we sum the effects of all neighbors by integrating over the volume from the radius of closest approacy, $r=2 a$, to infinity. Here also, $2 \pi \nu \tau_{c}$ is much smaller than one for all molecules close enough to have an appreciable effect, so that we have for the relaxation rate due to the neighbors $\left(1 / T_{1}\right)_{n}$,

$$
\begin{aligned}
\left(\frac{1}{T_{1}}\right)_{n}=\frac{3}{5} \gamma^{4} \hbar^{2} N_{0} \int_{2 a}^{\infty} \frac{3}{r^{6}} \cdot \frac{r^{2}}{12 D} & \cdot 4 \pi r^{2} d r \\
& =\frac{3 \pi}{10} \frac{\gamma^{4} h^{2} N_{0}}{a D} .
\end{aligned}
$$

$N_{0}$ in Eq. (49) is the number of molecules per $\mathrm{cm}^{3}$. We can make use of the well-known relation developed by Stokes, $D=k T / 6 \pi \eta a$, to write Eq. (49) as

$$
\left(1 / T_{1}\right)_{n}=9 \pi^{2} \gamma^{4} \hbar^{2} \eta N_{0} / 5 k T .
$$

Even when an experimental value of $D$ is available, the use of the Stokes relation represents no real sacrifice in accuracy, for the distance of closest approach, $a$, can at best be estimated only roughly.

Substituting numbers into (50) we obtain for water at $20^{\circ} \mathrm{C},\left(1 / T_{1}\right)_{n}=0.10 \mathrm{sec}^{-1}$. The relaxation time $T_{1}$ is then found by combining both effects: $\left(1 / T_{1}\right)=\left(1 / T_{1}\right)_{p}+\left(1 / T_{1}\right)_{n}=0.19$ sec. $^{-1}$ $+0.10 \mathrm{sec}^{-1}$, or $T_{1}=3.4 \mathrm{sec}$.

The effect of the partner thus appears to be somewhat more important than that of the neighboring molecules. We have probably overestimated the effect of the neighbors by neglecting the rotation of the nearest molecules as well as the effect upon the local field produced by them of the rotation of the molecule containing the proton $i$. That is, the appropriate $\tau_{c}$ for the nearest neighbors should be somewhat smaller than Eq. (48) would predict. Such refinements are hardly warranted in view of the obvious shortcomings of the model.

The experimentally determined value of $T_{1}$ for distilled water at $20^{\circ} \mathrm{C}$ is $2.3 \pm 0.5$ seconds, obtained by the direct method described in Section IV. The theoretical estimate of $T_{1}$ is satisfactorily close to the experimental value. Unfortunately, the possibility that the experimental value was influenced somewhat by dissolved oxygen cannot be excluded. From subsequent results with paramagnetic solutions, it is estimated that a saturation concentration of $\mathrm{O}_{2}$ could have contributed at most $0.3 \mathrm{sec}^{-1}$ to $\left(1 / T_{1}\right)$.

\section{EXPERIMENTAL RESULTS FOR VARIOUS LIQUIDS; COMPARISON WITH THEORY}

A more significant test of the theory is provided by measurements of the proton relaxation time in a series of similarly constituted liquids of widely differing viscosity. The conspicuous role of the viscosity in our description of the relaxation process will already have been noticed. Despite the crudeness of a description of the details of the molecular motion in terms of the macroscopic viscosity, it can hardly be doubted that there should be a close connection between $\eta$ and $\tau_{c}$. Table I lists the values of $T_{1}$ obtained for a series of hydrocarbons. These values, and all relaxation times quoted hereafter, were deter- 
mined by running a saturation curve on the sample, the direct measurements of $T_{1}$ for the water sample, and for the $0.002 \mathrm{NCuSO}_{4}$ solution (see Section IV) serving as a calibration of the circuit. The viscosity of each sample was measured by conventional methods and is listed for comparison. The wide range over which a rough proportionality exists between $\eta$ and $\left(1 / T_{1}\right)$ is striking. A more heterogeneous list of substances is given in Table II. A probable explanation of the exceptional behavior of sulfuric acid is the low density of protons in the material, or, more precisely, a relatively large value of the shortest proton-proton distance (the other constituents being non-magnetic).

A wide range in viscosity is covered by waterglycerin solutions. Figure 11 shows the variation of $T_{1}$ with viscosity for the water-glycerine system at room temperature. The viscosity values were obtained from tables in this case. Exact proportionality between $\left(1 / T_{1}\right)$ and $\eta$ is hardly to be expected for the progressive change in the character of the substance must bring with it a change in the environment of each proton and in the details of the local molecular motion. Actually a 1000 -fold change in $\eta$ is accompanied by a change of only 100 -fold in $T_{1}$.

A better experiment is one in which the same substance is used throughout, its viscosity being varied over the widest possible range by changing the temperature. Ethyl alcohol and glycerine are convenient for this purpose; each was studied over the temperature range between $60^{\circ} \mathrm{C}$ and $-35^{\circ} \mathrm{C}$. The temperature of the substance was measured with a copper-constantan thermocouple brought into the sample a short distance away from the $r-f$ coil. The temperature of the whole enclosure containing the coil and sample was established by circulating a suitable fluid at a controllable rate. The temperature of the

TABLE I. Relaxation time $T_{1}$, for protons in various hydrocarbons at $20^{\circ} \mathrm{C}$ and $29 \mathrm{Mc} / \mathrm{sec}$.

\begin{tabular}{lcc}
\hline & $\begin{array}{c}\text { Viscosity } \\
\text { (centipoises) }\end{array}$ & $\begin{array}{c}T_{1} \\
\text { (seconds) }\end{array}$ \\
\hline Petroleum ether & 0.48 & 3.5 \\
Ligroin & 0.79 & 1.7 \\
Kerosene & 1.55 & 0.7 \\
Light machine oil & 42 & 0.075 \\
Heavy machine oil & 260 & 0.013 \\
Mineral oil & 240 & 0.007 \\
\hline
\end{tabular}

sample could be held constant within $0.5^{\circ}$ while data for a saturation curve were taken. The viscosity values were taken from LandoltBörnstein, Physikalish-Chemische Tabellen.

The results obtained with ethyl alcohol at two frequencies are shown in Fig. 12, in the form of a logarithmic plot of the relaxation time $T_{1}$ against the ratio of the viscosity to the absolute temperature, $\eta / T$. According to our theory $\tau_{c}$, and hence $\left(1 / T_{1}\right)$, so long as $2 \pi \nu \tau_{c} \ll 1$, should be proportional to $\eta / T$, as indicated by the line drawn on the graph with slope -1 . Moreover, the relaxation time should be independent of $\nu$, the radiofrequency. Both predictions are confirmed by the experimental results.

A much wider range in $\eta / T$ is accessible with a pure glycerine sample. Glycerine, as is well known, ordinarily becomes super-cooled down to temperatures far below its freezing point of $18^{\circ} \mathrm{C}$, and at the lowest temperatures prevailing in the experiment the liquid was nearly glass-like. The glycerine results are plotted in Fig. 13 which includes also the results of line-width measurements in the low temperature region. The characteristic time $T_{2}$ associated with the line broadening is obtained by setting $\gamma \Delta H=2 / T_{2}$, where $\Delta H$ is the observed interval in gauss between points of maximum slope on the absorption curve. This procedure, as explained earlier, would be correct for a Gaussian line shape.

In the region of low viscosity the $T_{1}$ values for glycerine display the behavior encountered in alcohol, namely, inverse proportionality between $T_{1}$ and $\eta / T$, and independence of $T_{1}$ and $\nu$. The new feature is the minimum in $T_{1}$, followed by increasing $T_{1}$ as $\eta$ continues to increase. The inverse line-width $T_{2}$, on the other hand, decreases monotonically in the region where it could be measured, with perhaps a slight tendency to flatten off at the highest $\eta / T$ values. The curves

TABLE II. Relaxation time $T_{1}$, for protons in various polar liquids at $20^{\circ} \mathrm{C}$ and $29 \mathrm{Mc} / \mathrm{sec}$.

\begin{tabular}{lcc}
\hline \hline & $\begin{array}{c}\text { Viscosity } \\
\text { (centipoises) }\end{array}$ & $\begin{array}{c}T_{1} \\
\text { (seconds) }\end{array}$ \\
\hline Diethyl ether & 0.25 & 3.8 \\
Water & 1.02 & 2.3 \\
Ethyl alcohol & 1.2 & 2.2 \\
Acetic acid & 1.2 & 2.4 \\
Sulfuric acid & 25 & 0.7 \\
Glycerine & 1000 & 0.023 \\
\hline \hline
\end{tabular}




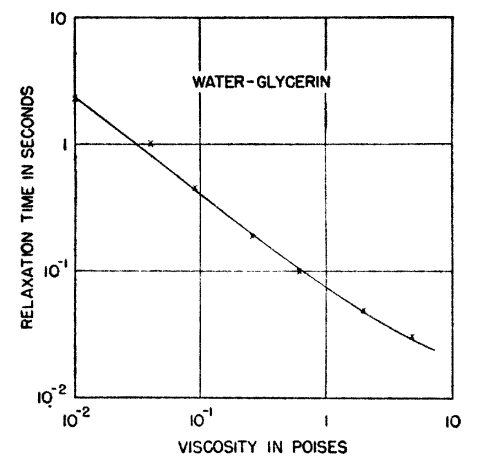

FIG. 11. Relaxation time for protons in water-glycerin solutions, measured at $29 \mathrm{Mc} / \mathrm{sec}$.

drawn on the graph are based on the theory, which must now be applied to this more general case, the restriction $2 \pi \nu \tau_{c} \ll 1$ being lifted.

The discussion will be simplified by the assumption that $\tau_{c}$, at a given temperature, is the same for all sources of the local field. We write Eq. (46) in the form:

$$
\frac{1}{T_{1}}=C_{1}\left[\frac{\tau_{e}}{1+\omega^{2} \tau_{c}^{2}}+\frac{2 \tau_{e}}{1+4 \omega^{2} \tau_{c}^{2}}\right]
$$

where $C_{1}$ includes the factors which are independent of the temperature and frequency. (The variation of $b$ and $a$ with temperature is a negligible effect.) The asymptotic behavior of $T_{1}$ is simple: for $\omega \tau_{c} \ll 1,\left(1 / T_{1}\right) \approx 3 C_{1} \tau_{c}$; for $\omega \tau_{c} \gg 1$, $\left(1 / T_{1}\right) \approx 3 C_{1} /\left(2 \omega^{2} \tau_{c}\right)$. In the intermediate region a minimum in $T_{1}$ occurs for $\omega \tau_{c}=1 / \sqrt{2}$, with $T_{1 \text { (min) }}=3 \omega /\left(2^{1} C_{1}\right)$. If we may assume that $\tau_{c}$ is proportional to $\eta / T$, the shape of the curve $\log T_{1}$ vs. $\log (\eta / T)$ should be the same for all substances to which the theory applies; changes in $C_{1}$ or in the factor connecting $\tau_{c}$ and $\eta / T$ merely shift the curve. Two such curves have been drawn on the graph in Fig. 13.

The salient features of the experimental results are well reproduced, although there are quantitative discrepancies. The horizontal shift of the minimum is somewhat less than the ratio of the frequencies, and the experimental minimum is flatter than predicted. This may be blamed in part upon experimental inaccuracy, but it must be remembered that Eq. (51) neglects the variation in $\tau_{c}$ among the neighbors. As we saw earlier, a proper treatment of the neighbors introduces a distribution of relaxation times, which

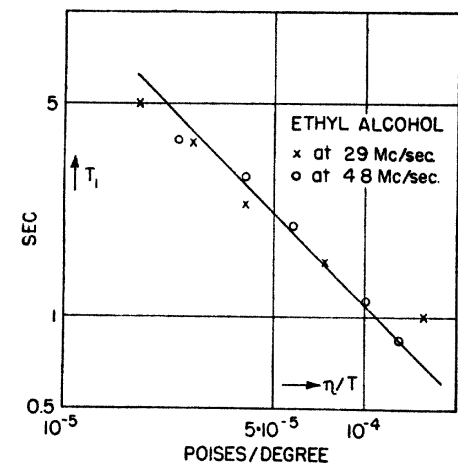

FIG. 12. Relaxation time for protons in ethyl alcohol, measured at $29 \mathrm{Mc} / \mathrm{sec}$. and at $4.8 \mathrm{Mc} / \mathrm{sec}$.

would have the effect of flattening the minimum in the $T_{1}$ curve.

To discuss the line-width parameter, $T_{2}$, we go back to Eq. (35) which determines $T_{2}^{\prime}$ and insert for $J_{0}(\nu),\left(4 / 5 b^{6}\right) 2 \tau_{c} /\left(1+\omega^{2} \tau_{c}^{2}\right)$. We thus obtain, making use of the constant $C_{1}$ of Eq. (51),

$$
\left(1 / T_{2}{ }^{\prime}\right)^{2}=(3 / \pi) C_{1} \tan ^{-1}\left(2 \tau_{c} / T_{2}{ }^{\prime}\right) .
$$

For sufficiently large values of $\tau_{c}, T_{2}{ }^{\prime}$ attains the value $\left(2 / 3 C_{1}\right)^{\frac{1}{2}}$, which is identical with $T_{2}{ }^{\prime \prime}$, the time characterizing the spin-spin coupling in the rigid lattice. When $\tau_{c}$ is small, we have, from (52), $\left(1 / T_{2}{ }^{\prime}\right) \approx(6 / \pi) C_{1} \tau_{c}$. The dashed line in Fig. 13 has been drawn according to this relation in a region in which the contribution of $\left(1 / T_{1}\right)$ to the line width is negligible. The agreement with experiment is satisfactory.

To the left of the minimum in $T_{1}$ we should have $\left(1 / T_{1}\right)=3 C_{1} \tau_{c}$ so that in this region, according to Eq. (38),

$$
\begin{aligned}
& \left(1 / T_{2}\right)=(2 / \pi)^{\frac{1}{2}}\left(1 / T_{2}{ }^{\prime}\right)+\left(1 / 2 T_{1}\right) \\
& \quad=3 C_{1} \tau_{c}\left[(2 / \pi)^{\frac{1}{3}}+\frac{1}{2}\right]=1.01 / T_{1} .
\end{aligned}
$$

This result signifies only that $T_{1}$ and $T_{2}$ are approximately equal when $2 \pi \nu \tau_{c} \ll 1$.

The general features of the simplified theory are presented in Fig. 14. The $T_{2}$ curve is located by the value of $T_{2}{ }^{\prime \prime}$, which depends on the spin-spin interaction in the "frozen" substance, according to Eq. (25). The break between the sloping and the horizontal part of the $T_{2}$ curve occurs near $\tau_{c}=T_{2}{ }^{\prime \prime} / \sqrt{2}$, according to (52). The minimum in the $T_{1}$ curve is located by $\omega \tau_{c}=1 / \sqrt{2}$, and thus both curves are fixed. That the transition regions occupy, in most cases, rather small 
ranges on the logarithmic plot is a consequence of the weakness of the spin-spin interaction compared to $\gamma H_{0} \hbar$.

We can now understand why the true line width in many liquids is unobservably small. The rapid fluctuations in the perturbing fields very nearly average out; the intensity in the local field spectrum is no greater at $\nu=0$ than it is at $\nu=\nu_{0}$, resulting in the near equality of $T_{1}$ and $T_{2}$ to the left of the $T_{1}$ minimum. But if $T_{2}$ is of the order of one second, as $T_{1}$ is in many cases, the corresponding line width is of the order of $10^{-4}$ gauss. To observe this width, the applied field $H_{0}$ would have to be homogeneous over the sample to less than 1 part in $10^{8}$.

As $\tau_{c}$ increases the line width increases, eventually becoming observable and finally attaining its asymptotic value. A striking qualitative demonstration of this effect is obtained by allowing molten paraffin to solidify in the sample tube while observing the proton resonance on the oscilloscope. If the field is reasonably homogeneous, a pronounced broadening of the line is observed when the paraffin freezes.

\section{RELAXATION TIME AND LINE WIDTH IN SOLUTIONS CONTAINING PARA- MAGNETIC IONS}

The addition of paramagnetic ions to water, as was first shown by Bloch, Hansen, and Packard, ${ }^{3}$ can influence markedly the proton relaxation time. The magnetic moment of such an ion is of the order of one Bohr magneton, and the interaction energy $\mu_{\text {ion }} \mu_{p} / r^{3}$ is thus some $10^{3}$ times larger than that of the interactions previously considered. Nevertheless, the discussion of the last section can be adapted to this case without much modification. The problem is essentially that treated in estimating the effect of the neighboring molecules. The appropriate value of $\tau_{c}$ is determined in the same way, with one possible exception to be mentioned later, and will not be much different in magnitude, so that we again have $2 \pi \nu \tau_{c} \ll 1$. Equation (50), adapted to this case, then reads:

$$
\begin{aligned}
\left(1 / T_{1}\right)=2 \pi \gamma^{2} \mu_{\text {eff }}^{2} N_{\text {ion }} / 5 a D & =12 \pi^{2} \gamma^{2} \eta N_{\text {ion }} \mu_{\text {eff }}^{2} / 5 k T .
\end{aligned}
$$

In general, the value of $\mu_{\text {eff }}{ }^{2}$ can be determined from data on paramagnetic susceptibility. Stokes' relation has again been used to express $a D$ in terms of $\eta / k T$; any difference between the mobility of an ion and that of a water molecule is thereby neglected. $N_{\text {ion }}$ is the number of ions per $\mathrm{cm}^{3}$.

According to Eq. (54) the relaxation time should be inversely proportional to the ionic concentration and to the square of the magnetic moment of the paramagnetic ion. The experimental results presented in Fig. 15 confirm the first prediction, except for a marked and unexplained deviation at very low concentrations.

The predicted proportionality between $\left(1 / T_{1}\right)$ and $\mu_{\text {eff }}{ }^{2}$ is tested by the data in Table III. The values of $\mu_{\text {off }}$ in the middle column were derived from measurements of $T_{1}$ in paramagnetic solutions of known concentration by assuming arbitrarily the value $2.0 \mathrm{Bohr}$ magnetons for $\mathrm{Cu}^{++}$. These numbers are to be compared with the last column which contains the values of $\mu_{\text {eff }}$ derived from susceptibility measurements. It is not surprising that in the cases of $\mathrm{Ni}^{++}, \mathrm{Co}^{++}$, and $\mathrm{Fe}(\mathrm{CN})_{6}$ - the $\mu_{\text {eff }}$ obtained from the nuclear relaxation measurements is too small, for there are important contributions from non-diagonal elements to the magnetic moment of these ions..$^{23}$ Indeed, $\mathrm{Co}^{++}$and $\mathrm{Fe}(\mathrm{CN})_{0}-$ show strong deviations from Curie's law. Non-diagonal elements would give rise to high frequency perturbations which would be ineffective in producing nuclear relaxation.

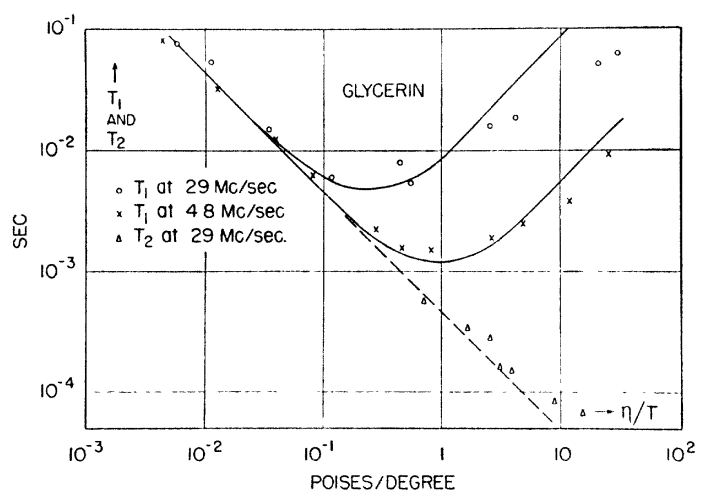

FIg. 13. The relaxation time $T_{1}$ and the line-width parameter $T_{2}$, plotted against the ratio of viscosity to absolute temperature, for glycerin.

${ }^{23}$ G. J. Gorter, Paramagnetic Relaxation (Elsevier Publishing Company, Inc., New York, 1947), p. 4. Also, J. H. Van Vleck, Electric and Magnetic Susceptibilities (Oxford University Press, New York, 1932), Chapter XI. 


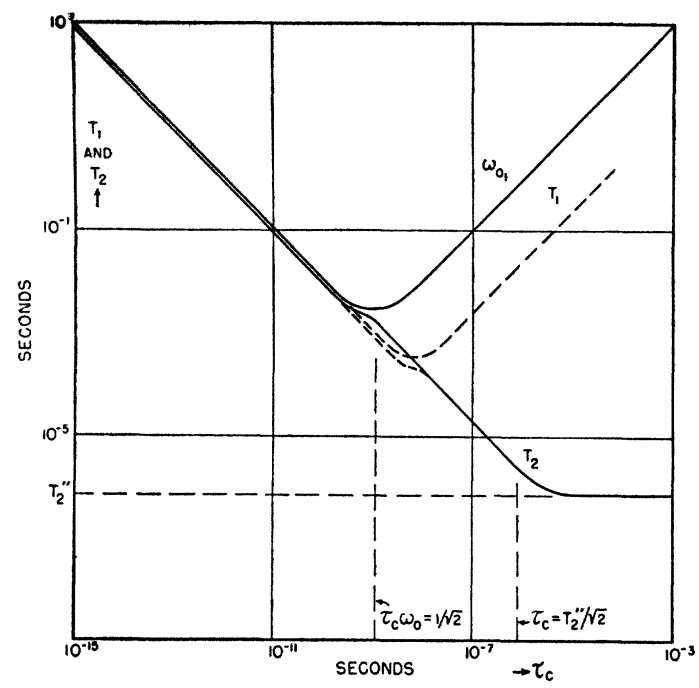

FIG. 14. Dependence of $T_{1}$ and $T_{2}$ upon $\tau_{c}$, according to the simplified theory in which all interactions are assumed to have the same correlation time.

The magnitude of $T_{1}$ as predicted by Eq. (54) agrees well with experiment. In the case of $\mathrm{Fe}^{+++}$, for example, Eq. (54) with $\eta=0.01, \mu_{\text {ion }}=5.9$ Bohr magnetons, yields $T_{1}=0.08$ second, for a concentration of $1018 / \mathrm{cm}^{3}$. The value observed at this concentration is 0.06 second. Such close agreement must be regarded as accidental.

An effect which has not been taken into account in the above discussion is the relaxation of the electron spins (i.e., paramagnetic relaxation) which may in some cases limit the value of $\tau_{c}$. Paramagnetic relaxation times as short as $10^{-9}$ second have been observed in crystals at room temperature. ${ }^{24}$ Some measurements of paramagnetic dispersion in solutions; reported by Zavoisky, ${ }^{25}$ indicate relaxation times of the order of $10^{-8}$ to $10^{-9}$ second. In applying Eq. (54) we have tacitly assumed that the paramagnetic relaxation time, under the conditions of our experiment was not much shorter than $10^{-11}$ second. It will be interesting to examine nuclear relaxation induced by paramagnetic ions under conditions for which the characteristic time $\tau_{c}$ due to thermal motion is quite long. The actual $\tau_{c}$ may then be determined by the paramagnetic relaxation effect.

The line width in paramagnetic solutions is of

${ }^{24}$ R. L. Cummerow, D. Halliday, and G. E. Moore, Phys. Rev. 72, 1233 (1947).

${ }^{25}$ E. Zavoisky, J. Phys. USSR 9, 211 (1945). particular interest, for the extremely short spinlattice relaxation time observed in concentrated solutions permits a test of the relation between $T_{1}$ and $T_{2}$. As we have seen, the theory predicts that $T_{1}$ and $T_{2}$ should be about equal when $2 \pi \nu \tau_{c} \ll 1$. That this is indeed the case can be seen by comparing Fig. 15 with Fig. 16, which gives the measured line width, expressed in terms of $T_{2}$, for highly concentrated $\mathrm{Fe}^{+++}$ solutions.

\section{NUCLEAR RELAXATION EXPERIMENTS INVOLVING $\mathrm{F}^{19}, \mathrm{Li}^{7}$, AND $\mathrm{D}^{2}$}

Several additional experiments on liquids will be reported briefly. The resonances of both $\mathrm{H}^{1}$ and $\mathrm{F}^{19}$ were studied in the liquid $\mathrm{CHFCl}_{2}$, a "Freon," which was condensed in a glass tube and sealed off. Both lines were narrow and the intensities were nearly equal, as would be expected. (The spin of $F^{19}$ is $\frac{1}{2}$, and its gyromagnetic ratio only a few percent less than that of the proton.) The measured relaxation times were $3.0 \mathrm{sec}$. and $2.6 \mathrm{sec}$. for $\mathrm{H}^{1}$ and $\mathrm{F}^{19}$, respectively. According to the theory these times should be equal, for the dominant effect should be the $\mathrm{H}^{1}-\mathrm{F}^{19}$ interaction within the molecule, and the product $\gamma_{\mathrm{H}^{1}}{ }^{2} \gamma_{\mathrm{F}^{192}}$ occurs in the generalized form of Eq. (34) in either case. Within the experimental error this is confirmed.

The compound $\mathrm{BeF}_{2}$ combines with water in any proportion. The behavior of both the proton and the fluorine resonances in $\mathrm{BeF}_{2} \cdot x \mathrm{H}_{2} \mathrm{O}$ was found to resemble that of the proton resonance in glycerine. With increasing viscosity (decreasing $x$ ) $T_{1}$ decreases to a minimum value of about $10^{-3}$ second, thereafter increasing to a final value of 0.2 second in pure $\mathrm{BeF}_{2}$, an amorphous glassy solid. The line width meanwhile increases from an unobservably small value to approximately 10 gauss in the anhydrous solid.

The resonance absorption of $\mathrm{Li}^{7}$ was examined at $14.5 \mathrm{Mc} / \mathrm{sec}$. in solutions of $\mathrm{LiCl}$ and $\mathrm{LiNO}_{3}$, with the results given in Table IV. The addition of paramagnetic ions affects the proton relaxation time more drastically than it does that of $\mathrm{Li}^{7}$, which is probably to be explained by the mutual repulsion of the positive ions, or by the existence of a "shield" of hydration around the $\mathrm{Li}$ ion. In the absence of paramagnetic ions the lithium relaxation time exceeds that of the protons, but 
not by as large a factor as the simplified theory would predict. This may be attributable to the effect of nuclear quadrupole interaction, a phenomenon which is much more conspicuous in the following example.

The deuteron and proton resonances were examined, at the same frequency, in a sample of 50 percent heavy water. The relaxation times observed for $\mathrm{D}^{2}$ and $\mathrm{H}^{1}$ were 0.5 and 3.0 sec., respectively. Contrary to what would be expected from the ratio of the magnetic moments, the deuteron relaxation time is the shorter. However, the interaction of the electric quadrupole moment of the deuteron with a fluctuating inhomogeneous electric field can bring about thermal relaxation. We omit the analysis of this process, which parallels closely the treatment of dipoledipole interaction, and point out only that the interaction energy, in order of magnitude, is $e Q\left(\partial \mathcal{E}_{z} / \partial z\right)$, where $Q$ is the nuclear quadrupole moment in $\mathrm{cm}^{2}$; this is to be compared with $\mu H_{\text {loc }}$. If the fluctuation spectrum of $\partial \mathcal{E}_{z} / \partial z$ is assumed similar to that of $H_{\text {loc }}$, the observed value of $T_{1}$ for the deuteron in our experiment can be accounted for by assuming a reasonable value of $\partial \mathcal{E}_{z} / \partial z$ in the $\mathrm{D}_{2} \mathrm{O}$ or HDO molecule. $\llbracket \uparrow \uparrow$ The quadrupole interaction is probably less effective in the case of the lithium ion because the immediate surroundings of the ion are electrically more symmetrical.

\section{NUCLEAR RELAXATION IN HYDROGEN GAS}

We have reported earlier ${ }^{26}$ the observation of nuclear resonance absorption in hydrogen gas at room temperature, at pressures between 10 and 30 atmospheres. The low density of nuclei severely limited the accuracy of measurement, but it was possible to ascertain that the relaxation time at 10 atmospheres was approximately 0.015 second. $T_{1}$ appeared to increase with increasing pressure. The width of the line was less than 0.15 gauss.

The interpretation of these results is closely related to our theory of relaxation in liquids. In each case the key to the problem is the observation that the local field fluctuates at a

\footnotetext{
IT A treatment of relaxation by quadrupole coupling will be found in the Thesis to be presented by one of the authors (N.B.) at the University of Leiden.

${ }^{26}$ E. M. Purcell, $\cdot$ R. V. Pound, and N. Bloembergen, Phys. Rev. 70, 986 (1946).
}

TABLE III. Comparison of magnetic moments of ions inferred from nuclear relaxation measurements and from static susceptibility measurements.

\begin{tabular}{lcc}
\hline \hline Ion & $\begin{array}{c}\text { meff in Bohr } \\
\text { magnetons } \\
\text { from nuclear } \\
\text { relaxation } \\
\text { experiments) }\end{array}$ & $\begin{array}{c}\text { meff in Bohr } \\
\text { magnetons } \\
\text { (from } \\
\text { susceptibility } \\
\text { measurements)* }\end{array}$ \\
\hline $\mathrm{Er}^{+++}$ & 8.5 & 9.4 \\
$\mathrm{Fe}^{+++}$ & 5.5 & 5.9 \\
$\mathrm{Cr}^{+++}$ & 4.1 & 3.8 \\
$\mathrm{Cu}^{+++}$ & 2.0 & 1.9 \\
$\mathrm{Ni}^{++}$ & 1.8 & 3.2 \\
$\mathrm{Co}$ & 1.1 & $4.5-5.3$ \\
$\mathrm{Fe}(\mathrm{CN})_{8}---$ & 0.1 & 2.4 \\
\hline
\end{tabular}

* The numbers in this column are those listed by Gorter, reference 23, pp. 14 and 15, with the exception of the value for $\mathrm{Fe}(\mathrm{CN}) \mathrm{C}^{--}$ which is based on the measurements of L. C. Jackson, Proc. Phys. Soc. 50, 707 (1938)

rate much higher than $\nu_{0}$. There are two distinct local magnetic fields at the position of one proton in the $\mathrm{H}_{2}$ molecule. One arises from the rotation of the molecule as a whole; the other originates, as in the water molecule, in the magnetic moment of the other proton in the molecule. The corresponding interaction energies are fairly large and have been accurately measured in molecular beam experiments ${ }^{27}$ where the interaction causes a splitting of the proton resonance into six components, in the lowest rotational state of ortho-hydrogen. The interactions involve the rotational quantum number $J$ and its spatial quantization $m_{J}$. In a gas at ordinary pressures, $m_{J}$, and, to a lesser extent, $J$, may be expected to change frequently as a result of molecular collisions. If $m_{J}$ were to change with each colli-

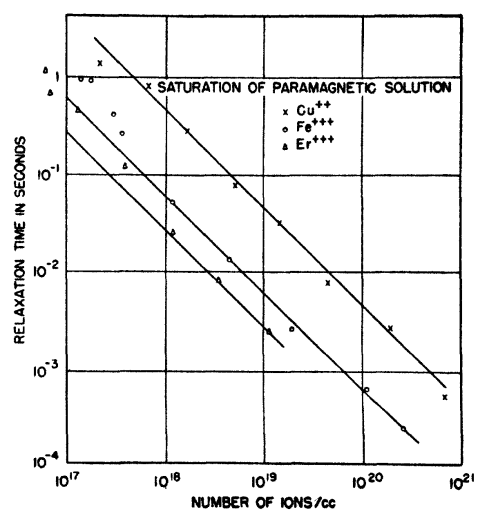

FIG. 15. Relaxation time $T_{1}$, for solutions of paramagnetic ions, measured at $29 \mathrm{Mc} / \mathrm{sec}$.

${ }^{27}$ J. M. B. Kellogg, I. I. Rabi, N. F. Ramsey, and J. R. Zacharias, Phys. Rev. 57, 677 (1940). 
TABLE IV.

\begin{tabular}{|c|c|c|c|}
\hline \multirow[b]{2}{*}{ Substance } & \multirow{2}{*}{$\left(\frac{\text { gram atom } \mathrm{H}}{\text { gram atom } \mathrm{Li}}\right)$} & \multicolumn{2}{|c|}{$\begin{array}{l}\text { Relaxation time } \\
\text { in sec. }\end{array}$} \\
\hline & & for $\mathrm{Li}^{7}$ & for $\mathrm{H}^{1}$ \\
\hline $\begin{array}{l}\mathrm{LiCl}+\mathrm{H}_{2} \mathrm{O} \\
\mathrm{LiNO}{ }_{3}+\mathrm{H}_{2} \mathrm{O} \\
\mathrm{LiNO}_{3}+\mathrm{H}_{2} \mathrm{O}+\mathrm{Fe}\left(\mathrm{NO}_{3}\right)_{2} \\
\mathrm{LiCl}+\mathrm{H}_{2} \mathrm{O}+\mathrm{CrCl}_{3} \\
\mathrm{LiCl}+\mathrm{H}_{2} \mathrm{O}+\mathrm{CuSO} 4\end{array}$ & $\begin{array}{l}5.8 \\
14 \\
8 \\
6.5 \\
6.6\end{array}$ & $\begin{array}{l}1.75 \\
2.7 \\
0.11 \\
0.24 \\
0.18\end{array}$ & $\begin{array}{l}0.4 \\
1.1 \\
0.0023 \\
0.0095 \\
0.013\end{array}$ \\
\hline
\end{tabular}

sion the correlation time for the local fields would be the mean time between collisions, which in hydrogen at 10 atmospheres is approximately $10^{-11}$ second. The situation is thus similar to that in a liquid of low viscosity, in that $2 \pi \nu \tau_{c} \ll 1$. We should therefore be prepared to find (a) that $T_{1} \propto 1 / \tau_{c} ;(\mathrm{b})$ that $T_{1}$ and $T_{2}$ are about equal, and very much larger than the reciprocal of the frequency splitting observed in the molecular beam experiment. Since we have identified $\tau_{c}$ with the time between collisions, $T_{1}$ should increase with increasing pressure, as observed. The implication of (b) is that the line should be very narrow, as observed, and that the line width should vary inversely with the pressure. This last effect would have been masked by inhomogeneities in the magnetic field, but the experimental results on closely analogous cases in liquids leave little room for doubt that "pressure narrowing" can occur.

The effect of the magnetic fields associated with neighboring molecules is negligible except at extremely high pressures, and in this respect the theoretical analysis is less uncertain than it was in the case of the liquids. We omit the derivation and present only the result, which was

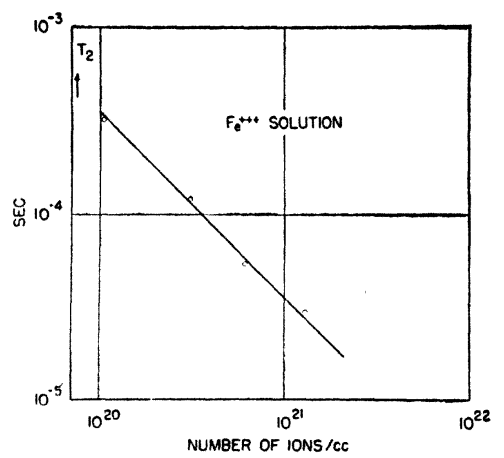

FIG. 16. The line-width parameter $T_{2}$, determined from measurements of line width in solutions containing a high concentration of ferric ions. first obtained by J. S. Schwinger, and can be reached by a process similar to that followed in Section VII.

$$
\begin{aligned}
& \frac{1}{T}=2 \tau_{\mathrm{c}} \gamma^{2}\left[\frac{1}{3} H^{\prime 2} J(J+1)\right. \\
&\left.+3 H^{\prime \prime 2} \frac{J(J+1)}{(2 J-1)(2 J+3)}\right] .
\end{aligned}
$$

The field at one proton due to the molecular rotation is specified by means of the constant $H^{\prime}$ in Eq. (55), which has the value 27 gauss. $^{27}$ $H^{\prime \prime}$ is a measure of the field at one proton due to the other and has the value 34 gauss. At room temperature we have to do mainly with the state $J=1$; molecules with $J=0,2$, etc., (para-hydrogen) are, of course, inert in the experiment. Identifying $\tau_{c}$ with the mean time between collisions calculated from gas kinetic data we find, using Eq. (55), $T_{1}=0.03$ seconds, at 10 atmospheres, in satisfactory agreement with experiment. The theoretical value will be reduced if $m_{J}$ does not change in every collision, as assumed.

\section{NUCLEAR RELAXATION IN ICE}

The process which we have invoked to explain nuclear relaxation in liquids has no counterpart in an ideal crystal. Nevertheless, many solids show evidence, in the form of specific heat anomalies or dielectric dispersion, of internal degrees of freedom other than vibration. We shall discuss only one example, which we have studied experimentally in some detail, that of ice.

Ice shows a marked dielectric dispersion at rather low frequencies. The residual entropy of ice also provides evidence for internal freedom which has been attributed ${ }^{28}$ to the two available positions for the proton in the $\mathrm{O}-\mathrm{H}-\mathrm{O}$ bond. Without inquiring into the connection between these two phenomena, or into the details of the internal motion, we might assume that our parameter $\tau_{c}$ is proportional to the Debye characteristic time $\tau_{D}$, deduced from dielectric dispersion data. The nuclear relaxation data for protons in ice have been plotted against $\tau_{D}$ in Figs. 17 and 18 , the value of $\tau_{D}$ for each temper-

${ }^{28}$ Linus Pauling, The Nature of the Chemical Bond (Cornell University Press, Ithaca, New York, 1940), p. $302 \mathrm{ff}$. 


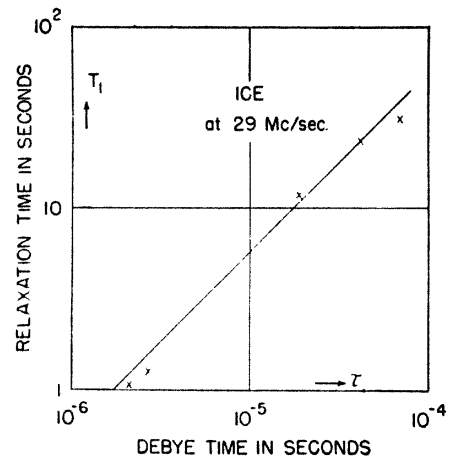

FIg. 17. Proton relaxation time in ice, plotted against the Debye relaxation time derived from dielectric dispersion data.

ature having been taken from the measurements of Wintsch. ${ }^{21}$ Figure 17 shows $T_{1}$ for ice, in the temperature range $-2{ }^{\circ} \mathrm{C}$ to $-40^{\circ} \mathrm{C}$. It is clear that we have to do with the ascending branch of the typical $T_{1}$ curve, and that the assumption $\tau_{c} \approx \tau_{D}$ was not a bad one. The line width increases as the temperature is lowered, as shown by the results plotted in Fig. 18. The curve in Fig. 18 was calculated by means of Eq. (52). It appears that $T_{2}$ is approaching, at the lowest temperatures, its asymptotic value. The limit approached by the line width is indicated as about 16 gauss, which is in good agreement with the value calculated from the crystal structure of ice, assuming the nuclei at rest.

It is interesting to note that although the relaxation time $T_{1}$, for water at $20^{\circ} \mathrm{C}$, is about equal to that of ice at $-5^{\circ} \mathrm{C}$, the two cases represent opposite extremes of behavior. In water $2 \pi \nu \tau_{c} \ll 1$, and the line is narrow; in ice $2 \pi \nu \tau_{c} \gg 1$, and the line is broad.

\section{CONCLUDING REMARKS}

We have been able to account for the line widths and spin-lattice relaxation times observed in a number of liquids, in paramagnetic solutions, in hydrogen gas, and in ice. In particular, the extremely small width of the resonance line in ordinary liquids, and the accompanying inverse dependence of $T_{1}$ upon the viscosity of the liquid, appear as consequences of a situation in which all perturbing fields fluctuate in a random manner at a rate much higher than the nuclear Larmor frequency - a situation without an obvious parallel in atomic or molecular spectroscopy.

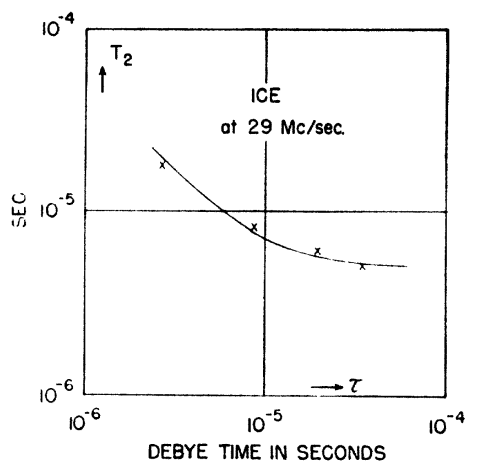

FIG. 18. $T_{2}$ for ice, plotted against the Debye relaxation time.

The quantitative agreement is as good as we could have expected in view of the simplifying assumptions which were necessary in applying the theory to a particular substance, and the considerable uncertainty in the absolute magnitude of the experimental relaxation times. Not all of the features of the theory are thereby tested; for example, we cannot infer from the experimental data on water the relative magnitude of the inter- and intramolecular effects which were treated separately in Section X. Possibly with improvements in the accuracy of measurement of relaxation times such points could be tested. On the other hand, further refinement of the theory, in the direction of a more faithful representation of the molecule and its immediate environment, would appear to be neither easy nor, at present, very profitable, except perhaps in the case of a structure like that of ice which is well determined.

Since rotational and translational degrees of freedom play such an important role in determining line widths and relaxation times, the study of nuclear resonances provides another means for investigating those phenomena in solids which have been ascribed to the onset, or cessation, of a particular type of internal motion. Experiments of this sort have already been reported by Bitter and collaborators. ${ }^{29}$

An understanding of the factors controlling $T_{1}$ and $T_{2}$ should be helpful in planning experiments to detect nuclear resonances and to measure nuclear gyromagnetic ratios. Both $T_{1}$ and

${ }^{29}$ F. Bitter, N. L. Alpert, H. L. Poss, C. G. Lehr, and S. T. Lin, Phys. Rev. 71, 738 (1947); N. L. Alpert, Phys. Rev. 72, 637 (1947). 
$T_{2}$ must be taken into account in estimating the sensitivity attainable.

Finally, we want to emphasize that our understanding of the main features of the spinlattice relaxation process does not extend to tightly bound crystalline structures in which, presumably, only vibrational degrees of freedom are active. We have observed in a fluorite crystal at room temperature a relaxation time of about 9 seconds. This alone is surprising, for the Waller theory applied to this case predicts a relaxation time of the order of one hour. A more remarkable result is that reported by Rollin and Hatton, ${ }^{5}$ who find that the relaxation time in lithium fluoride remains of the order of seconds down to $2^{\circ} \mathrm{K}$.

\section{ACKNOWLEDGMENT}

The authors have benefited from numerous illuminating and stimulating discussions with Professor H. C. Torrey of Rutgers University, Professor J. H. Van Vleck, Professor J. S. Schwinger, and with Professor C. J. Gorter of the University of Leiden, Visiting Lecturer at Harvard in the summer of 1947.

The work described in this paper was supported by a Frederick Gardner Cottrell Special Grant-in-Aid from the Research Corporation.

\section{APPENDIX}

The intensity of the radiofrequency signal resulting from nuclear paramagnetic resonance absorption varies over a very wide range depending on the various parameters descriptive of the sample. For a large signal such as that produced by the protons in water, little attention need be given to the detection technique. Other samples often require extreme care lest a very small signal be lost in noise. To see what experimental conditions are imposed by the nature of the sample it is useful to develop a formula that expresses the signal-to-noise ratio as a function of the parameters descriptive of the apparatus and of the sample.

It might be well to point out that, though here we work entirely with the nuclear absorption, the detectability of the nuclear dispersion, observed with an apparatus of the bridge type when the residual signal is due to a phase unbalance, is almost exactly the same. As shown by Bloch, ${ }^{6}$ the main difference between the two effects is that the signal caused by the dispersion effect increases asymptotically to a maximum as the r-f field $H_{1}$ is increased, whereas for the signal caused by the absorption there is an optimum value for $H_{1}$. As a result, one of the effects may be the more useful for some purposes and the other for others. For conducting a search for unknown lines, an apparatus using the dispersion effect might be best since the search need not be made to cover a range of values of $r$-f field strength and modulation amplitude. For investigating line shape and relaxation phenomena the absorption effect gives results that are probably easier to interpret.

In all experimental arrangements so far employed the sample is contained in a resonant circuit, most of ten in the coil of a shunt resonant coil and condenser. In Section I it was shown that a quantity, called there the $Q$ of the absorption, was given by

$$
1 / Q \equiv \delta=\gamma^{2} h^{2} N_{0} I(I+1) \nu_{0} T_{2}{ }^{*} / 3 k T .
$$

This quantity is the ratio of the energy lost per radian from the $r-f$ field to the system of nuclear spins to the energy stored in the oscillating $r-f$ field. Thus it is the same kind of a quantity as the ordinary $Q$ used as a figure of merit for simple coils and condensers. The effect of the nuclear resonance on the circuit is, then, to reduce the $Q$ of the circuit from $Q_{0}$ to a value $Q_{r}$ given by

$$
1 / Q_{r}=\left(1 / Q_{0}\right)+\zeta \delta
$$

The quantity $\zeta$ is a filling factor for the circuit determined by the fraction of the total $r-f$ magnetic energy that is actually stored in the space occupied by the sample.

To obtain the largest signal for detection with a circuit of a given $Q$, it is now apparent that the addition of the loss attributable to $\delta$ should produce the largest possible change in the voltage across the tuned circuit. This is achieved when the generator driving the circuit is of the constant current type, or, in other words, if the generator has a very high internal impedance compared to the impedance of the tuned circuit at resonance. To achieve this the very small coupling condensers are used on the input sides of the circuits described in Section II. 
Consider the use of a modulated magnetic field. At times when the field is such that the nuclear resonance does not occur the voltage across the resonant circuit is proportional to $Q_{0}$. At the peak of the resonance, the voltage is proportional to $Q_{r}$ which, since $\delta$ is very small compared to $1 / Q_{0}$, may be written

$$
Q_{r}=Q_{0}\left(1-\zeta \delta Q_{0}\right) .
$$

The resulting modulated voltage may be expanded in Fourier series if the shape of the absorption line is known, the signal indicating the presence of nuclear absorption being contained in the sidebands. For observation of the effect on an oscillograph all of these should be taken into account but for a system using the "lock-in" amplifier, sensitive only in the immediate neighborhood of a single frequency, only a single pair of sidebands differing in frequency from the driving frequency by just the modulation frequency need be considered. The signalto-noise ratio computed from these also gives an estimate of the usefulness of an oscillograph as an indicator, if the noise bandwidth of the system is suitably chosen.

The voltage across the tuned circuit may be considered to be given by

$$
\begin{aligned}
E=\left(2 R P_{0}\right)^{\frac{1}{2}}\left[\cos \omega_{0} t+\frac{1}{4} A \zeta \delta Q_{0} \cos \left(\omega_{0}+\omega_{m}\right) t\right. & \left.+\frac{1}{4} A \zeta \delta Q_{0} \cos \left(\omega_{0}-\omega_{m}\right) t\right],
\end{aligned}
$$

where $R$ is the shunt resistance of the resonant circuit, $P_{0}$ is the power dissipated in the resonant circuit, $\omega_{0}$ is $2 \pi$ times the radiofrequency, $\omega_{m}$ is $2 \pi$ times the modulation frequency, and $A$ is a constant, of the order of unity, that depends on the exact shape of the line, the amount of the modulation swing and the shape of the modulating wave. Since this voltage is for the unloaded circuit, the power available as a signal in the sidebands is

$$
P_{s}=P_{0}\left(A \zeta \delta Q_{0}\right)^{2} / 32 \text {. }
$$

It follows from the analysis, in Section IV, of the saturation effect that there is an optimum strength of the driving signal field $H_{\mathrm{i}}$, given by

$$
\left(\gamma H_{1}\right)^{2} T_{1} T_{2}{ }^{*}=1 \text {. }
$$

For this magnitude of the driving signal the value of $\delta$ is reduced to one-half its value in Eq. (56) because of partial saturation. Remem- bering that $H_{1}$ is one-half the amplitude of the oscillating field, Eq. (59) may be used to compute the optimum value for $P_{0}$, giving

$$
P_{0}=\omega_{0} V_{c} / 2 \pi \gamma^{2} T_{1} T_{2}{ }^{*} Q_{0},
$$

where $V_{c}$ is an effective volume for the part of the circuit in which magnetic energy is stored. For a simple solenoid this is very nearly the volume inside the coil, since the field outside is weak, and the energy is proportional to the square of the field. This equation may be put into many other forms. One useful form is

$$
P_{0}=H_{0} H_{2} V_{c} / 2 Q_{0} T_{1},
$$

where $\mathrm{H}_{2}$ is the full width in gauss of the absorption line between points of half the peak absorption, assuming the damped-oscillator line shape. This shows that for lines having widths determined by inhomogeneity in the magnetic field, the input power required to produce saturation depends on none of the properties of the sample except $T_{1}$. Putting Eq. (60) into Eq. (58) for $P_{0}$ and using one-half the value of $\delta$ given by Eq. (56), one gets, for the total signal power available,

$$
\begin{aligned}
P_{s}=V_{c} Q_{0}(A \zeta)^{2} h^{4} N_{0}{ }^{2} \gamma^{2} \nu_{0}{ }^{3} T_{2}{ }^{*} & \\
& \times[I(I+1)]^{2} / 1152 T_{1}(k T)^{2} .
\end{aligned}
$$

If the detection apparatus is described by an effective noise band width $2 B$ ( $B$ being the band width at the indicating instrument) and an effective over-all noise figure, ${ }^{30} \mathrm{~F}$, the resulting signal-to-noise power ratio is

$$
\begin{aligned}
P_{s} / P_{n}= & V_{c} Q_{0}(A \zeta)^{2} h^{4} N_{0}{ }^{2} \gamma^{2} \nu_{0}^{3} T_{2}{ }^{*} \\
& \times[I(I+1)]^{2} / 2304(k T)^{2}(k T B F) T_{1} .
\end{aligned}
$$

The two factors containing $k T$ are kept separate to emphasize that the noise figure of the detector is not independent of the temperature. If $F$ is large, most of the noise originates in the apparatus and not in the circuit connected to its input terminals.

In most cases the output indicator will respond linearly to the signal and noise amplitudes and, thus, the ratio of the response to the absorption line to the r.m.s. noise fluctuation is

$$
\begin{aligned}
A_{s} / A_{n}=V_{c}^{\frac{1}{2}} Q_{0}^{\frac{1}{3}} A \zeta h^{2} N_{0} \gamma \nu_{0}^{\frac{1}{3}} T_{2}{ }^{* \frac{1}{2}} I \\
\times(I+1) / 48 k T(k T B F)^{\frac{1}{3}} T_{1^{\frac{1}{3}} .}
\end{aligned}
$$

${ }^{30}$ H. T. Friis, Proc. I.R.E. 32, 419 (1944). 
To demonstrate completely the dependence on the frequency and on the dimensions of the circuit an empirical relation showing the dependence of $Q_{0}$ on these quantities may be inserted. Thus

$$
Q_{0}=0.037 \nu_{0}^{\frac{1}{2}} V_{c}^{\frac{1}{3}},
$$

where $\nu_{0}$ is to be measured in c.p.s. and $V_{c}$ in $\mathrm{cm}^{3}$ may be used. The numerical constant is based on experimental values of $Q$ for coils of about $1-\mathrm{cm}^{3}$ volume in the region of $30 \mathrm{Mc} / \mathrm{sec}$.

Often the frequency of operation is determined by the magnetic field available. In such a case, using Eq. (63) for $Q_{0}$, Eq. (62) becomes

$$
\begin{aligned}
A_{s} / A_{n} \approx & \left.1.62 \times 10^{-4} V_{c}^{3}\right\} A h^{2} N_{0} \gamma^{11 / 4} H_{0}{ }^{7 / 4} \\
& \times T_{2}{ }^{*} I I(I+1) / k T(k T B F)^{1} T_{1}^{1} .
\end{aligned}
$$

Observe that the signal-to-noise amplitude ratio is proportional to $\gamma^{11 / 4}$, for a given field, concentration, volume of the circuit, filling factor for the circuit, and ratio of $T_{1} / T_{2}{ }^{*}$. The quantity $V_{c} \zeta N_{0}$ is the total number of nuclei present. Thus Eq. (64) shows that the signal-to-noise ratio is inversely proportional to $V_{c}^{t}$ for a given number of nuclei and for all the other quantities constant. This favors as high a concentration as possible. Note, however, that if the sample is a crystal with a rigid lattice so that $T_{2}{ }^{*}$ is determined by the static dipole-dipole coupling, it is preferable to have the nuclei spread out. In this event, the signal-to-noise ratio is proportional to $V_{c}^{1 / 6}, \zeta$ and the total number of nuclei in the sample being held constant. This is true only if, as the concentration is reduced, interactions other than the spin-spin interaction among the nuclei under investigation remain negligible.

The signals produced by the nuclei in liquids, if the relaxation time $T_{1}$ can be made so short as to be an important contributant to the line width, are particularly strong. This condition can be obtained by the use of solutions containing paramagnetic ions to shorten $T_{1}$ sufficiently to make $T_{2}{ }^{*} / T_{1} \approx 1$. For this case, Eq. (64) may be written as,

$$
\begin{aligned}
A_{\varepsilon} / A_{n} \approx 1.2 \times 10^{-26} N H_{0}{ }^{7 / 4} \mu^{11 / 4} & \\
& \times(I+1) / V_{c}^{1} B^{1} I^{7 / 4},
\end{aligned}
$$

where $\mu$ is the magnetic moment in nuclear magnetons. This gives a signal-to-noise ratio of $1.4 \times 10^{6}$ for the protons in a $1-\mathrm{cm}^{3}$ water sample with a magnetic field of 15,000 gauss and a band width of 1 c.p.s. Thus even with an oscillograph and a high modulation frequency, requiring a noise band width of perhaps $5 \mathrm{kc} / \mathrm{sec}$., a signal of the order of $2 \times 10^{4}$ times the r.m.s. amplitude of noise may be expected. On the other hand, a 1-molar solution of nuclei having magnetic moments of 0.1 nuclear magnetons produces a signal-to-noise ratio of only about 1.3 when a $1-\mathrm{cm}^{3}$ sample in a 15,000-gauss field is used with an apparatus having a 1-c.p.s. band width. For this magnetic field, band width, and volume of circuit, about $10^{18}$ nuclei having spins of $\frac{1}{2}$ and magnetic moments of one nuclear magneton would be required to give a signal equal to noise. 


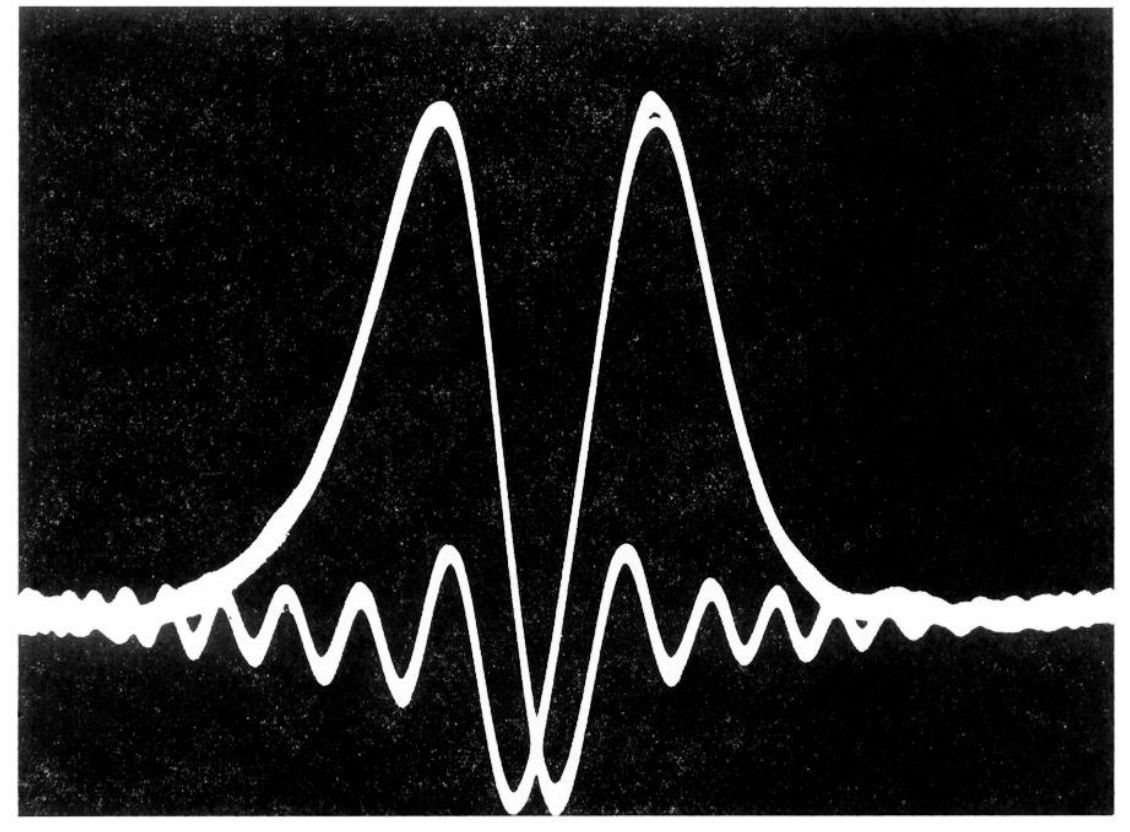

FIg. 10. An example of the transient effect described in Section IV, observed in water at $29 \mathrm{Mc} / \mathrm{sec}$. The field modulation and the oscilloscope sweep are both sinusoidal, but there is a small phase difthere is a small phase dif-
ference between them. The wiggles occur after the magnetic field has passed through resonance. 


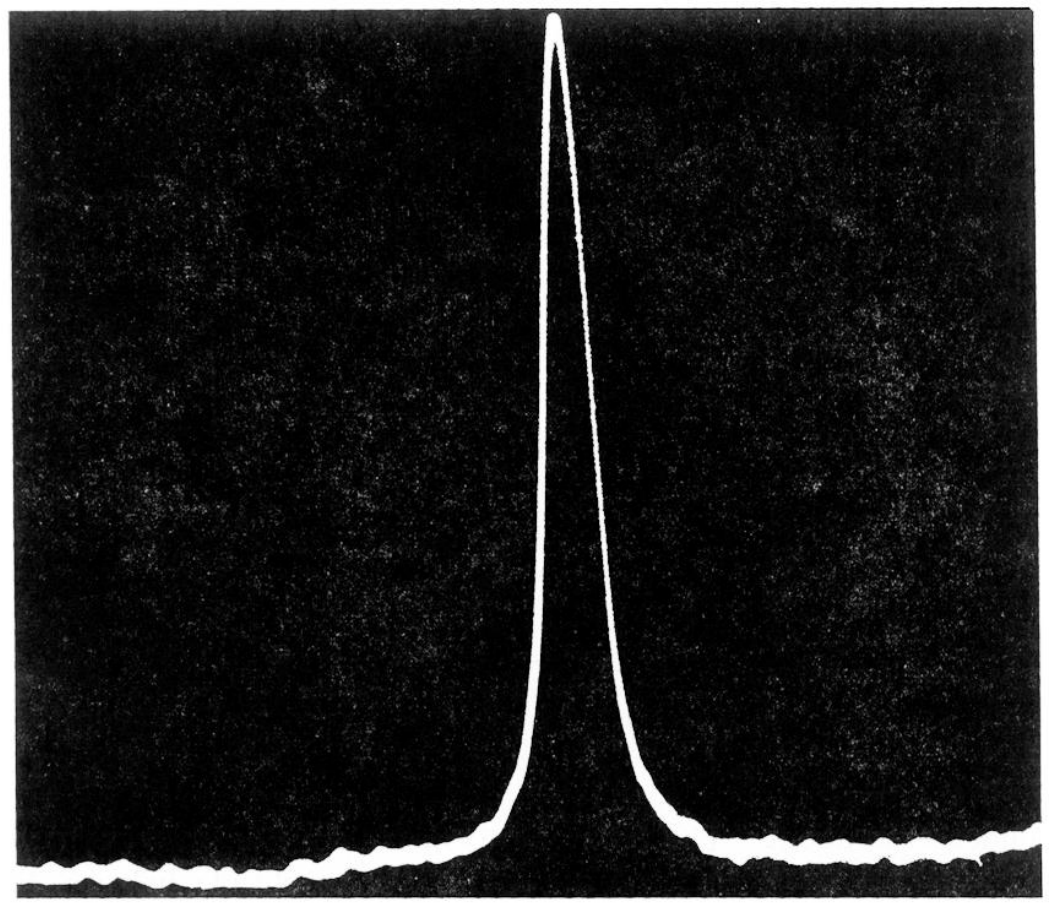

FIg. 3. Proton resonance (absorption curve) in ferric nitrate solution. 
FIG. 4. Proton resonance (dispersion curve) in ferric nitrate solution

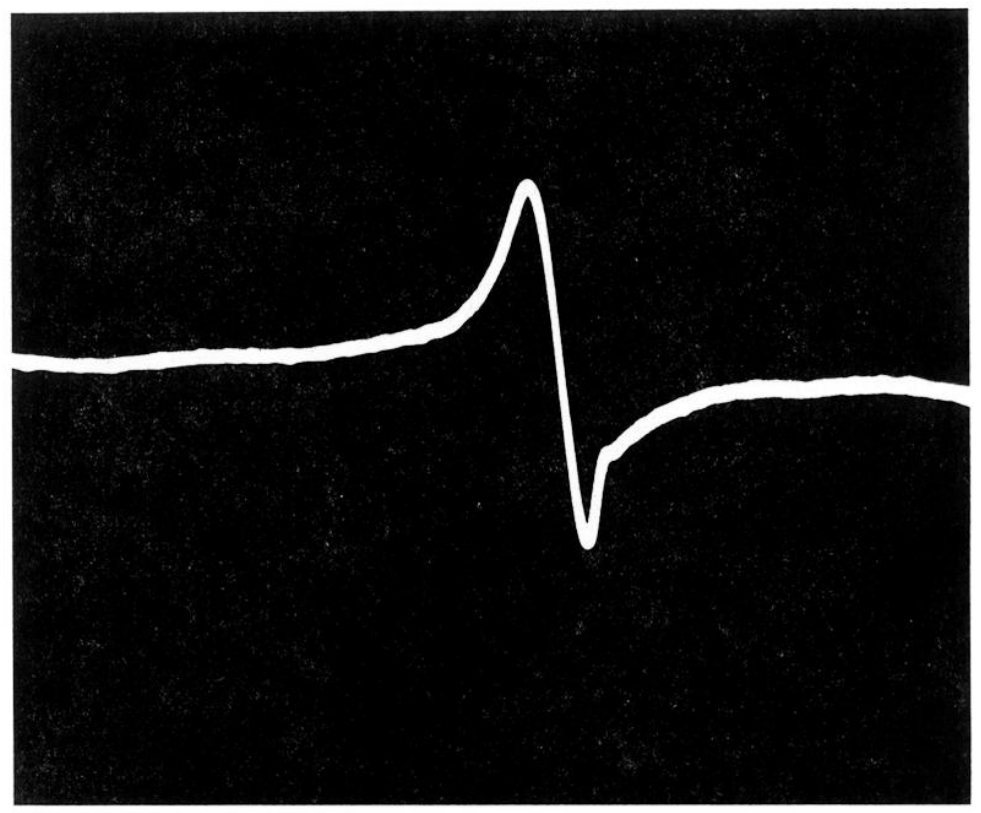

